Article

\title{
Generalization of the Geometry of Cathelineau Infinitesimal and Grassmannian Chain Complexes
}

\author{
Muhammad Khalid ${ }^{1, *}$, Azhar Iqbal ${ }^{2}$ and Javed Khan ${ }^{1}$ \\ 1 Department of Mathematical Sciences, Federal Urdu University of Arts, Science \& Technology, Karachi-75300, \\ Pakistan. \\ 2 Department of Basic Sciences, Dawood University of Engineering \& Technology, Karachi-74800, Pakistan. \\ * Correspondence: khalidsiddiqui@ fuuast.edu.pk
}

\begin{abstract}
In this article, a generalization of the geometry of Grassmannian chain complex of free abelian groups generated by the projective configuration of points and Cathelineau's infinitesimal complex of polylogarithmic groups is proposed. Firstly, homomorphisms for weight $n=2$ up to weight $n=6$ will be introduced to connect sub-complexes of Grassmannian and Cathelineau. Lately, generalization of these morphisms will be shown for weight $n=N$. The associated diagrams will also be proven to be commutative and bi-complex.
\end{abstract}

$7 \quad$ Keywords: homomorphism; Grassmannian; generalized geometry; cathelineau's complex

MSC: 19L20, 22E10, 11G55

\section{Introduction}

Grassmannian chain complex of free abelian groups generated by the projective configurations of points was first introduced by Suslin [1]. Suslin used two type of differential homomorphisms $d$ and $p$ to connect these free abelian groups. In Grassmannian chain complex each square is commutative and the composition of two same differential morphisms is zero [1]. Classical polylogarithmic functions had studied for many hundred years, first defined by Leibniz. Dilogarithm appear in the work of Spence, Abel, Kummer, Lobachesky, Hill, Roger, and Ramanujan etc but most important was the functional equation known as Abel's five term relations. Trilogarithms and its group $\mathcal{B}_{3}(F)$ was first introduced by Goncharov using generalized triple cross ratio of six points. Goncharov also generalized polylogarithmic group as $\mathcal{B}_{n}(F)$ and generalized Bloch-Suslin complex known as Goncharov's complex. Homomorphisms between Grassmannian and Bloch-Suslin complexes for Di-logarithm weight $n=2$ was defined by Goncharov [2-4]. Goncharov proved that the associated digram is bi-complex and commutative. Goncharov [2] also uses the duality of configurations in order to prove (projected seven-term) functional equation for the trilogarithmic group $\mathcal{B}_{3}(F)$ and verifies that a Complex forms among Grassmannian and Goncharov's Complexes in weight 3 is commutative. Cathelineau [5-7] defined analogy of Goncharov's complexes in the additive (both infinitesimal and tangential) setting called Cathelineau's complexes.

Cathelineau defined F-vector space as $\beta_{2}(F)$, generated by four term relation and $\beta_{3}(F)$, generated by 22 term relations for his generalized chain complex. Siddiqui [8] found projected triple cross ratio and indicated that it should be written as the ratio of two projected cross-ratios. Siddiqui $[8,9]$ also introduced variant of Cathelineau's complexes in both infinitesimal and tangential setting and describe their relations through homomorphisms with Grassmannian chain complexes of the projective configurations for weight $n=2$. Author also found morphisms between Grassmannian complex and Variant of Cathelineau's infinitesimal complex for weight $n=3$ and show that the associated diagram is commutative and bi-complex. 


\subsection{Grassmannian Complex}

Consider a free abelian group $G_{m}(n)$ generated by $m$-vectors of dimension $n$. Following is the Grassmannian bicomplex

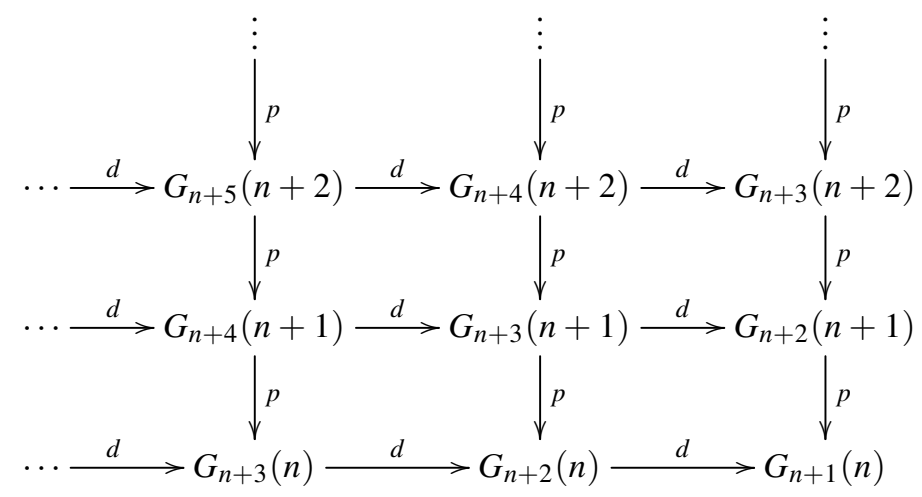

$d$ is called differential map given by

$$
d:\left(q_{0}, \ldots, q_{n}\right) \mapsto \sum_{i=0}^{n}(-1)^{i}\left(q_{0}, \ldots, \hat{q}_{i}, \ldots, q_{n}\right)
$$

and $p$ another differential morphism called projection morphism given by

$$
p:\left(q_{0}, \ldots, q_{n}\right) \mapsto \sum_{i=0}^{n}(-1)^{i}\left(q_{i} \mid q_{0}, \ldots, \hat{q}_{i}, \ldots, q_{n}\right)
$$

Lemma 1. The diagram (A) is bi-complex, i.e. $d \circ d=p \circ p=0$

Proof. For proof (see [1])

Lemma 2. The diagram (A) is commutative, i.e. $d \circ p=p \circ d$

Proof. For proof (see [1]) 


\subsubsection{Weight 3}

As defined in [2]

$$
r_{3}\left(q_{0}, \ldots, q_{6}\right)=\frac{\Delta\left(q_{0}, q_{1}, q_{3}\right) \Delta\left(q_{1}, q_{2}, q_{4}\right) \Delta\left(q_{2}, q_{0}, q_{5}\right)}{\Delta\left(q_{0}, q_{1}, q_{4}\right) \Delta\left(q_{1}, q_{2}, q_{5}\right) \Delta\left(q_{2}, q_{0}, q_{3}\right)}
$$

it is a triple cross ratio 6 points. Take $R_{3}(F) \subset Z\left[\mathbf{P}_{F}^{1} /\{0,1, \infty\}\right][2]$, defined as

$$
R_{3}(F)=\sum_{i=0}^{6}(-1)^{i} A l t_{6} r_{3}\left(q_{0}, \ldots, \hat{q}_{i}, \ldots, q_{6}\right)
$$

which is a seven term relation of triple ratio. Goncharov defines $\mathcal{B}_{3}(F)$, which is quotient subgroup $Z\left[\mathbf{P}_{F}^{1} /\{0,1, \infty\}\right] / R_{3}(F)$, the Goncharov's complex in weight $n=3$ is given by

$$
\mathcal{B}_{3}(F) \stackrel{\delta}{\longrightarrow} \mathcal{B}_{2}(F) \otimes F^{\times} \stackrel{\delta}{\longrightarrow} \wedge^{3} F^{\times}
$$

It is called cross ratio of four points. Define a map $\delta_{2}: Z\left[\mathbf{P}_{F}^{1} /\{0,1, \infty\}\right] \rightarrow \wedge^{2} F^{\times}$, defined as $[x] \rightarrow(1-x) \wedge x$, it has been proven that $\delta_{2}\left(R_{2}(F)\right)=0$ [2]. Define group $\mathcal{B}_{2}(F)$ the factor group of $Z\left[\mathbf{P}_{F}^{1} /\{0,1, \infty\}\right] / R_{2}(F)$. Now introduce Bloch-Suslin complex

$$
0 \stackrel{\delta}{\longrightarrow} \mathcal{B}_{2}(F) \stackrel{\delta}{\longrightarrow} \wedge^{2} F^{\times} \stackrel{\delta}{\longrightarrow} 0
$$

where

$$
r\left(q_{0}, q_{1}, q_{2}, q_{3}\right)=\frac{\Delta\left(q_{0}, q_{3}\right) \Delta\left(q_{1}, q_{2}\right)}{\Delta\left(q_{0}, q_{2}\right) \Delta\left(q_{1}, q_{3}\right)}
$$


63

\subsubsection{Weight $n$}

Goncharov [2] generalized the group $\mathcal{B}_{n}(F)=Z\left[\mathbf{P}_{F}^{1} /\{0,1, \infty\}\right] / R_{n}(F)$, where $R_{n}(F)$ is a kernel of the map $\delta_{n}: Z\left[\mathbf{P}_{F}^{1}\right] \rightarrow \mathcal{B}_{n-1}(F) \otimes F^{\times}$, so generalized Goncharov's complex is given as

$$
\mathcal{B}_{n}(F) \stackrel{\delta}{\rightarrow} \mathcal{B}_{n-1}(F) \otimes F^{\times} \stackrel{\delta}{\rightarrow} \mathcal{B}_{n-2}(F) \otimes \wedge^{2}(F) \stackrel{\delta}{\rightarrow} \ldots \stackrel{\delta}{\rightarrow} \mathcal{B}_{2}(F) \otimes \wedge^{n-2}(F) \stackrel{\delta}{\rightarrow} \frac{\wedge^{n} F^{\times}}{2-\text { torsion }}
$$

\subsection{Cathelineau's Complexes}

Cathlelineau [6] has defined the $F$ - Vector space which is an infinitesimal form of Bloch groups $\mathcal{B}_{n}(F)$ as follows

1. $\beta_{1}(F)=F$

2. $\beta_{2}(F)=\frac{F\left[F^{\bullet \bullet}\right]}{r_{2}(F)}$. where $r_{2}(F)$ is the kernel of $\partial_{2}: F\left[F^{\bullet \bullet}\right] \rightarrow F \otimes F^{\times}$defined by $[x] \rightarrow x \otimes x+(1-$ $x) \otimes(1-x)$. Cathelineau showed that $r_{2}(F)$ is a sub-vector space generated by four elements $[x]-[y]+$ $x\left[\frac{y}{x}\right]+(1-x)\left[\frac{1-y}{1-x}\right]$ therefore obtain a complex

$$
\beta_{2}(F) \stackrel{\partial}{\rightarrow} F \otimes_{F} F^{\times}
$$

where $\partial$ is an induced map defined as

$$
\partial:\langle x\rangle_{2} \mapsto x \otimes(x)+(1-x) \otimes(1-x)
$$

using tensor properties this map can be written as

$$
\partial:\langle x\rangle_{2} \mapsto x \otimes(x-1)-(1-x) \otimes(x)
$$

The functional equation in $\beta_{2}(F)$

1. A two term relation $\langle a\rangle_{2}=\langle 1-a\rangle_{2}$

2. An inversion relation $\langle a\rangle_{2}=-a\left\langle\frac{1}{a}\right\rangle_{2}$

3. A four term relation $\langle a\rangle_{2}-\langle b\rangle_{2}+a\left\langle\frac{b}{a}\right\rangle_{2}+(1-a)\left\langle\frac{1-b}{1-a}\right\rangle_{2}=0$

4. A distribution relation $\langle a\rangle_{2}^{m}=\sum_{\zeta^{m}=1} \frac{1-a^{m}}{1-a \zeta}\langle\zeta a\rangle_{2}$

If $r_{n}(F)$ is a kernel of the map defined as $\delta_{n}: F[F] \rightarrow \beta_{n-1} \otimes F^{\times} \oplus F \otimes \mathcal{B}_{n-1}(F)$ [6]. Now by taking $\beta_{n}(F)$ the factor group as

$$
\beta_{n}(F)=\frac{F\left[F^{\bullet \bullet}\right]}{r_{n}(F)}
$$

The Cathelineau chain complex [6] for groups $\beta_{n}(F)$ and $\mathcal{B}_{n}(F)$ is given as

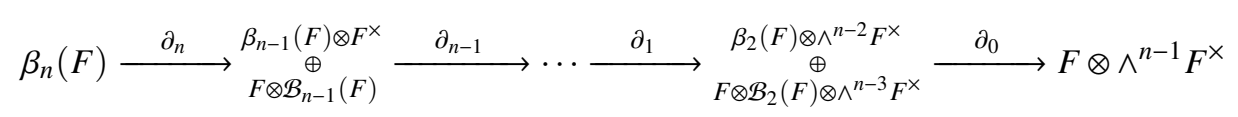

where $\partial_{n}$ is given by

$$
\partial_{n}:[r] \longmapsto\langle r\rangle_{n-1} \otimes r+(-1)^{n-1}(1-r) \otimes[r]_{n-1}
$$

Lemma 3. $\partial_{n-1} \circ \partial_{n}=0([6])$ 


\section{Geometry of Cathelineau and Grassmannian Complexes}

\section{3.1. Weight 2}

Construct the diagram of Grassmannian and Cathelineau infinitesimal complexes for weight $n=2$.

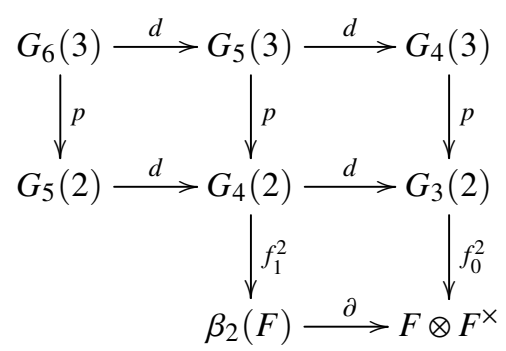

where

$$
f_{0}^{2}:\left(q_{0}, q_{1}, q_{2}\right) \rightarrow \sum_{i=0}^{2}(-1)^{i} \Delta\left(q_{0}, \ldots, \hat{q}_{i}, \ldots, q_{2}\right) \otimes \frac{\triangle\left(q_{0}, \ldots, \hat{q}_{i+1}, \ldots, q_{2}\right)}{\triangle\left(q_{0}, \ldots, \hat{q}_{i+2}, \ldots, q_{2}\right)} \quad(\bmod 3)
$$

and

$$
f_{1}^{2}\left(q_{0}, q_{1}, q_{2}, q_{3}\right)=\left\langle r\left(q_{0}, \ldots, q_{3}\right)\right\rangle_{2}
$$

Lemma 4. $f_{1}^{2}$ is independent of volume formation by vectors in $V_{2}$.

Proof. Let $f_{1}^{2}\left(q_{0}, q_{1}, q_{2}, q_{3}\right)$ can be written as

$$
f_{1}^{2}\left(q_{0}, q_{1}, q_{2}, q_{3}\right)=\left\langle\frac{\triangle\left(q_{0}, q_{3}\right) \triangle\left(q_{1}, q_{2}\right)}{\triangle\left(q_{0}, q_{2}\right) \triangle\left(q_{1}, q_{3}\right)}\right\rangle_{2}
$$

so by changing volume $V=\alpha V$ where $\alpha \in$ field $F$ then due to frictions the right side will remain unchanged, therefore $f_{1}^{2}$ is independent of volume form by vectors in $V_{2}$.

Lemma 5. $f_{1}^{2} \circ p$ is independent of length of vectors in $V_{2}$.

Proof. Let $f_{1}^{2} \circ p\left(q_{0}, q_{1}, q_{2}, q_{3}, q_{4}\right)$ can be written as

$$
f_{1}^{2} \circ p\left(q_{0}, q_{1}, q_{2}, q_{3}, q_{4}\right)=\sum_{i=0}^{4}\left\langle r\left(q_{i} \mid q_{0}, \ldots, \hat{q}_{i}, \ldots, q_{4}\right)\right\rangle_{2}
$$

so changing the length of vector like $\left(q_{0}, q_{1}, q_{2}, q_{3}, q_{4}\right)=\alpha\left(q_{0}, q_{1}, q_{2}, q_{3}, q_{4}\right)$ where $\alpha \in$ field $F$ then due to ratios the difference will be zero. Therefore $f_{1}^{2}$ is independent of length of vectors in $V_{2}$.

Lemma 6. $f_{0}^{2}$ is independent of volume form by vectors in $V_{2}$.

Proof. Let $f_{0}^{2}\left(q_{0}, q_{1}, q_{2}\right)$ can be written as

$$
f_{0}^{2}\left(q_{0}, q_{1}, q_{2}\right)=\frac{\Delta\left(q_{1}, q_{2}\right)}{\Delta\left(q_{0}, q_{2}\right)} \otimes \frac{\Delta\left(q_{0}, q_{2}\right)}{\Delta\left(q_{0}, q_{1}\right)}-\frac{\Delta\left(q_{0}, q_{1}\right)}{\Delta\left(q_{0}, q_{2}\right)} \otimes \frac{\Delta\left(q_{0}, q_{2}\right)}{\Delta\left(q_{1}, q_{2}\right)}
$$

${ }_{84}$ so if volume $V=\alpha V$ where $\alpha \in$ field $F$ then the right side will remain unchanged so $f_{0}^{2}$ is independent of volume form by vectors in $V_{2}$. 
86 Lemma 7. $f_{0}^{2} \circ p$ is independent of length of vectors in $V_{2}$.

\section{Proof.}

$$
\begin{aligned}
f_{0}^{2} \circ p\left(q_{0}, q_{1}, q_{2}, q_{3}\right)= & \frac{\Delta\left(q_{0}, q_{2}, q_{3}\right)}{\Delta\left(q_{0}, q_{1}, q_{3}\right)} \otimes \frac{\Delta\left(q_{0}, q_{1}, q_{3}\right)}{\Delta\left(q_{0}, q_{1}, q_{2}\right)}-\frac{\Delta\left(q_{0}, q_{1}, q_{2}\right)}{\Delta\left(q_{0}, q_{1}, q_{3}\right)} \otimes \frac{\Delta\left(q_{0}, q_{1}, q_{3}\right)}{\Delta\left(q_{0}, q_{2}, q_{3}\right)}- \\
& \frac{\Delta\left(q_{1}, q_{2}, q_{3}\right)}{\Delta\left(q_{1}, q_{0}, q_{3}\right)} \otimes \frac{\Delta\left(q_{1}, q_{0}, q_{3}\right)}{\Delta\left(q_{1}, q_{0}, q_{2}\right)}+\frac{\Delta\left(q_{1}, q_{0}, q_{2}\right)}{\Delta\left(q_{1}, q_{0}, q_{3}\right)} \otimes \frac{\Delta\left(q_{1}, q_{0}, q_{3}\right)}{\Delta\left(q_{1}, q_{2}, q_{3}\right)}+ \\
& \frac{\Delta\left(q_{2}, q_{1}, q_{3}\right)}{\Delta\left(q_{2}, q_{0}, q_{3}\right)} \otimes \frac{\Delta\left(q_{2}, q_{0}, q_{3}\right)}{\Delta\left(q_{2}, q_{0}, q_{1}\right)}-\frac{\Delta\left(q_{2}, q_{0}, q_{1}\right)}{\Delta\left(q_{2}, q_{0}, q_{3}\right)} \otimes \frac{\Delta\left(q_{2}, q_{0}, q_{3}\right)}{\Delta\left(q_{2}, q_{1}, q_{3}\right)}- \\
& \frac{\Delta\left(q_{3}, q_{1}, q_{2}\right)}{\Delta\left(q_{3}, q_{0}, q_{2}\right)} \otimes \frac{\Delta\left(q_{3}, q_{0}, q_{2}\right)}{\Delta\left(q_{3}, q_{0}, q_{1}\right)}+\frac{\Delta\left(q_{3}, q_{0}, q_{1}\right)}{\Delta\left(q_{3}, q_{0}, q_{2}\right)} \otimes \frac{\Delta\left(q_{3}, q_{0}, q_{2}\right)}{\Delta\left(q_{3}, q_{1}, q_{2}\right)}
\end{aligned}
$$

Proof. From the above diagram take

$$
G_{4}(3) \stackrel{p}{\longrightarrow} G_{3}(2) \stackrel{f_{0}^{2}}{\longrightarrow} F \otimes F^{\times}
$$

Assume that the four points $\left(q_{0}, q_{1}, q_{2}, q_{3}\right) \in G_{4}(3)$, apply map $p$ then $p\left(q_{0}, q_{1}, q_{2}, q_{3}\right)=\left(q_{0} / q_{1}, q_{2}, q_{3}\right)-$ $\left(q_{1} / q_{0}, q_{2}, q_{3}\right)+\left(q_{2} / q_{0}, q_{1}, q_{3}\right)-\left(q_{3} / q_{0}, q_{1}, q_{2}\right)$ now apply $f_{0}^{2}$, then

$$
\begin{aligned}
f_{0}^{2} \circ p\left(q_{0}, q_{1}, q_{2}, q_{3}\right)= & \Delta\left(q_{0}, q_{2}, q_{3}\right) \otimes \frac{\Delta\left(q_{0}, q_{1}, q_{3}\right)}{\Delta\left(q_{0}, q_{1}, q_{2}\right)}-\Delta\left(q_{0}, q_{1}, q_{3}\right) \otimes \frac{\Delta\left(q_{0}, q_{2}, q_{3}\right)}{\Delta\left(q_{0}, q_{2}, q_{1}\right)}+ \\
& \Delta\left(q_{0}, q_{1}, q_{2}\right) \otimes \frac{\Delta\left(q_{0}, q_{3}, q_{2}\right)}{\Delta\left(q_{0}, q_{3}, q_{1}\right)}-\Delta\left(q_{1}, q_{2}, q_{3}\right) \otimes \frac{\Delta\left(q_{1}, q_{0}, q_{3}\right)}{\Delta\left(q_{1}, q_{0}, q_{2}\right)}+ \\
& \Delta\left(q_{1}, q_{0}, q_{3}\right) \otimes \frac{\Delta\left(q_{1}, q_{2}, q_{3}\right)}{\Delta\left(q_{1}, q_{2}, q_{0}\right)}-\Delta\left(q_{1}, q_{0}, q_{2}\right) \otimes \frac{\Delta\left(q_{1}, q_{3}, q_{2}\right)}{\Delta\left(q_{1}, q_{3}, q_{0}\right)}+ \\
& \triangle\left(q_{2}, q_{1}, q_{3}\right) \otimes \frac{\Delta\left(q_{2}, q_{0}, q_{3}\right)}{\Delta\left(q_{2}, q_{0}, q_{1}\right)}-\Delta\left(q_{2}, q_{0}, q_{3}\right) \otimes \frac{\Delta\left(q_{2}, q_{1}, q_{3}\right)}{\Delta\left(q_{2}, q_{1}, q_{0}\right)}+ \\
& \triangle\left(q_{2}, q_{0}, q_{1}\right) \otimes \frac{\Delta\left(q_{2}, q_{3}, q_{1}\right)}{\Delta\left(q_{2}, q_{3}, q_{0}\right)}-\Delta\left(q_{3}, q_{1}, q_{2}\right) \otimes \frac{\Delta\left(q_{3}, q_{0}, q_{2}\right)}{\Delta\left(q_{3}, q_{0}, q_{1}\right)}+ \\
& \triangle\left(q_{3}, q_{0}, q_{2}\right) \otimes \frac{\Delta\left(q_{3}, q_{1}, q_{2}\right)}{\Delta\left(q_{3}, q_{1}, q_{0}\right)}-\Delta\left(q_{3}, q_{0}, q_{1}\right) \otimes \frac{\Delta\left(q_{3}, q_{2}, q_{1}\right)}{\Delta\left(q_{3}, q_{2}, q_{0}\right)} \\
& =0
\end{aligned}
$$

91 Lemma 9. The lower square of the diagram $D$ is commutative.

Proof. Let $\left(q_{0}, q_{1}, q_{2}, q_{3}\right) \in G_{4}(2)$. Apply morphism $d$, then

$$
d\left(q_{0}, q_{1}, q_{2}, q_{3}\right)=\left(q_{1}, q_{2}, q_{3}\right)-\left(q_{0}, q_{2}, q_{3}\right)+\left(q_{0}, q_{1}, q_{3}\right)-\left(q_{0}, q_{1}, q_{2}\right)
$$

now apply $f_{0}^{2}$, and get 24 terms, write them in the form of 12 terms as given below.

$$
f_{0}^{2} \circ d\left(q_{0}, \ldots, q_{3}\right)=\Delta\left(q_{2}, q_{3}\right) \otimes \frac{\Delta\left(q_{1}, q_{3}\right)}{\Delta\left(q_{1}, q_{2}\right)}-\Delta\left(q_{1}, q_{3}\right) \otimes \frac{\Delta\left(q_{2}, q_{3}\right)}{\Delta\left(q_{2}, q_{1}\right)}+\Delta\left(q_{1}, q_{2}\right) \otimes \frac{\Delta\left(q_{3}, q_{2}\right)}{\Delta\left(q_{3}, q_{1}\right)}-
$$




$$
\begin{aligned}
& \Delta\left(q_{2}, q_{3}\right) \otimes \frac{\Delta\left(q_{0}, q_{3}\right)}{\Delta\left(q_{0}, q_{2}\right)}+\Delta\left(q_{0}, q_{3}\right) \otimes \frac{\Delta\left(q_{2}, q_{3}\right)}{\Delta\left(q_{2}, q_{0}\right)}-\Delta\left(q_{0}, q_{2}\right) \otimes \frac{\Delta\left(q_{3}, q_{2}\right)}{\Delta\left(q_{3}, q_{0}\right)}+ \\
& \Delta\left(q_{1}, q_{3}\right) \otimes \frac{\Delta\left(q_{0}, q_{3}\right)}{\Delta\left(q_{0}, q_{1}\right)}-\Delta\left(q_{0}, q_{3}\right) \otimes \frac{\Delta\left(q_{1}, q_{3}\right)}{\Delta\left(q_{1}, q_{0}\right)}+\Delta\left(q_{0}, q_{1}\right) \otimes \frac{\Delta\left(q_{3}, q_{1}\right)}{\Delta\left(q_{3}, q_{0}\right)}- \\
& \Delta\left(q_{1}, q_{2}\right) \otimes \frac{\Delta\left(q_{0}, q_{2}\right)}{\Delta\left(q_{0}, q_{1}\right)}+\Delta\left(q_{0}, q_{2}\right) \otimes \frac{\Delta\left(q_{1}, q_{2}\right)}{\Delta\left(q_{1}, q_{0}\right)}-\Delta\left(q_{0}, q_{1}\right) \otimes \frac{\Delta\left(q_{2}, q_{1}\right)}{\Delta\left(q_{2}, q_{0}\right)}
\end{aligned}
$$

Take $\left(q_{0}, q_{1}, q_{2}, q_{3}\right) \in G_{4}(2)$ again, apply map $f_{1}^{2}$, then

$$
f_{1}^{2}\left(q_{0}, q_{1}, q_{2}, q_{3}\right)=\left\langle\frac{\Delta\left(q_{0}, q_{3}\right) \Delta\left(q_{1}, q_{3}\right)}{\Delta\left(q_{0}, q_{2}\right) \Delta\left(q_{1}, q_{3}\right)}\right\rangle_{2}
$$

now apply $\partial$

$$
\begin{aligned}
\partial \circ f_{1}^{2}\left(q_{0}, q_{1}, q_{2}, q_{3}\right)= & \frac{\Delta\left(q_{0}, q_{3}\right) \Delta\left(q_{1}, q_{3}\right)}{\Delta\left(q_{0}, q_{2}\right) \Delta\left(q_{1}, q_{3}\right)} \otimes\left(\frac{\Delta\left(q_{0}, q_{3}\right) \Delta\left(q_{1}, q_{3}\right)}{\Delta\left(q_{0}, q_{2}\right) \Delta\left(q_{1}, q_{3}\right)}-1\right)- \\
& \left(1-\frac{\Delta\left(q_{0}, q_{3}\right) \Delta\left(q_{1}, q_{3}\right)}{\triangle\left(q_{0}, q_{2}\right) \Delta\left(q_{1}, q_{3}\right)}\right) \otimes \frac{\Delta\left(q_{0}, q_{3}\right) \Delta\left(q_{1}, q_{3}\right)}{\Delta\left(q_{0}, q_{2}\right) \Delta\left(q_{1}, q_{3}\right)}
\end{aligned}
$$

using Siegel cross ratio properties [15] then

$$
\begin{aligned}
\partial \circ f_{1}^{2}\left(q_{0}, q_{1}, q_{2}, q_{3}\right)= & \frac{\Delta\left(q_{0}, q_{3}\right) \Delta\left(q_{1}, q_{3}\right)}{\Delta\left(q_{0}, q_{2}\right) \Delta\left(q_{1}, q_{3}\right)} \otimes \frac{\Delta\left(q_{0}, q_{1}\right) \Delta\left(q_{2}, q_{3}\right)}{\Delta\left(q_{0}, q_{2}\right) \Delta\left(q_{1}, q_{3}\right)}- \\
& \frac{\Delta\left(q_{0}, q_{1}\right) \Delta\left(q_{2}, q_{3}\right)}{\Delta\left(q_{0}, q_{2}\right) \Delta\left(q_{1}, q_{3}\right)} \otimes \frac{\Delta\left(q_{0}, q_{3}\right) \Delta\left(q_{1}, q_{3}\right)}{\Delta\left(q_{0}, q_{2}\right) \Delta\left(q_{1}, q_{3}\right)}
\end{aligned}
$$

after simplifications

$$
\begin{aligned}
\partial \circ f_{1}^{2}\left(q_{0}, \ldots, q_{3}\right)= & \Delta\left(q_{2}, q_{3}\right) \otimes \frac{\Delta\left(q_{1}, q_{3}\right)}{\Delta\left(q_{1}, q_{2}\right)}-\Delta\left(q_{1}, q_{3}\right) \otimes \frac{\Delta\left(q_{2}, q_{3}\right)}{\Delta\left(q_{2}, q_{1}\right)}+\Delta\left(q_{1}, q_{2}\right) \otimes \frac{\Delta\left(q_{3}, q_{2}\right)}{\Delta\left(q_{3}, q_{1}\right)}- \\
& \Delta\left(q_{2}, q_{3}\right) \otimes \frac{\Delta\left(q_{0}, q_{3}\right)}{\Delta\left(q_{0}, q_{2}\right)}+\Delta\left(q_{0}, q_{3}\right) \otimes \frac{\Delta\left(q_{2}, q_{3}\right)}{\Delta\left(q_{2}, q_{0}\right)}-\Delta\left(q_{0}, q_{2}\right) \otimes \frac{\Delta\left(q_{3}, q_{2}\right)}{\Delta\left(q_{3}, q_{0}\right)}+ \\
& \Delta\left(q_{1}, q_{3}\right) \otimes \frac{\Delta\left(q_{0}, q_{3}\right)}{\Delta\left(q_{0}, q_{1}\right)}-\Delta\left(q_{0}, q_{3}\right) \otimes \frac{\Delta\left(q_{1}, q_{3}\right)}{\Delta\left(q_{1}, q_{0}\right)}+\Delta\left(q_{0}, q_{1}\right) \otimes \frac{\Delta\left(q_{3}, q_{1}\right)}{\Delta\left(q_{3}, q_{0}\right)}- \\
& \Delta\left(q_{1}, q_{2}\right) \otimes \frac{\Delta\left(q_{0}, q_{2}\right)}{\Delta\left(q_{0}, q_{1}\right)}+\Delta\left(q_{0}, q_{2}\right) \otimes \frac{\Delta\left(q_{1}, q_{2}\right)}{\Delta\left(q_{1}, q_{0}\right)}-\Delta\left(q_{0}, q_{1}\right) \otimes \frac{\Delta\left(q_{2}, q_{1}\right)}{\Delta\left(q_{2}, q_{0}\right)}
\end{aligned}
$$

From Eq.(16) and Eq.(20), it is proved that the diagram D is commutative.

\subsection{Weight 3 (Trilogarithm)}

For this weight, connect the subcomplex of Cathelineau complex in weight 3 with the subcomplex of Grassmannian given as

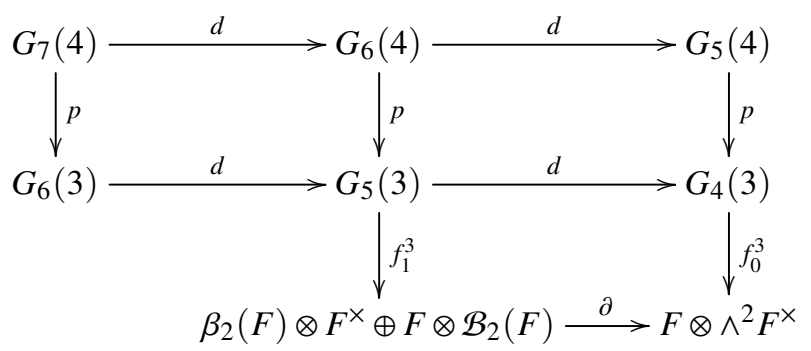


where

$$
f_{0}^{3}\left(q_{0}, \ldots, q_{3}\right)=\sum_{i=0}^{3}(-1)^{i} \Delta\left(q_{0}, \ldots, \hat{q}_{i}, \ldots, q_{3}\right) \otimes \frac{\Delta\left(q_{0}, \ldots, \hat{q}_{i+1}, \ldots, q_{3}\right)}{\Delta\left(q_{0}, \ldots, \hat{q}_{i+2}, \ldots, q_{3}\right)} \wedge \frac{\Delta\left(q_{0}, \ldots, \hat{q}_{i+2}, \ldots, q_{3}\right)}{\Delta\left(q_{0}, \ldots, \hat{q}_{i+3}, \ldots, q_{3}\right)} \quad(\bmod 4)
$$

and

$$
\begin{aligned}
f_{1}^{3}\left(q_{0}, \ldots, q_{4}\right) & =-\frac{1}{3}\left(\sum _ { i = 0 } ^ { 4 } ( - 1 ) ^ { i } \left(\left\langle r\left(q_{i} \mid q_{0}, \ldots, \hat{q}_{i}, \ldots, q_{4}\right)\right\rangle_{2} \otimes \prod_{\substack{j \neq i \\
j=0}}^{4} \Delta\left(q_{0}, \ldots, \hat{q}_{i}, \hat{q}_{j}, \ldots, q_{4}\right)\right.\right. \\
& \left.-\prod_{\substack{j \neq i \\
j=0}}^{4} \Delta\left(q_{0}, \ldots, \hat{q}_{i}, \hat{q}_{j}, \ldots, q_{4}\right) \otimes\left[q_{i} \mid q_{0}, \ldots, \hat{q}_{i}, \ldots, q_{4}\right]_{2}\right) \quad(\bmod 5)
\end{aligned}
$$

$94 \quad$ Lemma 10. $f_{0}^{3} \circ p=0$

Proof. Let $\left(q_{0}, q_{1}, q_{2}, q_{3}, q_{4}\right) \in G_{5}(4)$, apply map $p$

$$
p\left(q_{0}, q_{1}, q_{2}, q_{3}, q_{4}\right)=\sum_{i=0}^{4}(-1)^{i}\left(q_{i} \mid q_{0}, \ldots, \hat{q}_{i}, \ldots, q_{4}\right)
$$

On applying map $f_{0}^{3}$ on $p\left(q_{0}, q_{1}, q_{2}, q_{3}, q_{4}\right)$, then

$$
\begin{aligned}
f_{0}^{3} \circ p\left(q_{0}, \ldots, q_{4}\right) & =\frac{\Delta\left(q_{0}, q_{2}, q_{3}, q_{4}\right)}{\Delta\left(q_{0}, q_{1}, q_{3}, q_{4}\right)} \otimes \frac{\Delta\left(q_{0}, q_{1}, q_{3}, q_{4}\right)}{\Delta\left(q_{0}, q_{1}, q_{2}, q_{4}\right)} \wedge \frac{\Delta\left(q_{0}, q_{1}, q_{2}, q_{4}\right)}{\Delta\left(q_{0}, q_{1}, q_{2}, q_{3}\right)} \\
& -\frac{\Delta\left(q_{0}, q_{1}, q_{2}, q_{4}\right)}{\Delta\left(q_{0}, q_{1}, q_{2}, q_{3}\right)} \otimes \frac{\Delta\left(q_{0}, q_{2}, q_{3}, q_{4}\right)}{\Delta\left(q_{0}, q_{1}, q_{3}, q_{4}\right)} \wedge \frac{\Delta\left(q_{0}, q_{1}, q_{3}, q_{4}\right)}{\Delta\left(q_{0}, q_{1}, q_{2}, q_{3}\right)} \\
& \cdot \\
& \cdot \\
& -\frac{\Delta\left(q_{4}, q_{2}, q_{3}, q_{0}\right)}{\Delta\left(q_{4}, q_{1}, q_{3}, q_{0}\right)} \otimes \frac{\Delta\left(q_{4}, q_{1}, q_{3}, q_{0}\right)}{\Delta\left(q_{4}, q_{1}, q_{2}, q_{0}\right)} \wedge \frac{\Delta\left(q_{4}, q_{1}, q_{2}, q_{0}\right)}{\Delta\left(q_{4}, q_{1}, q_{2}, q_{3}\right)} \\
& +\frac{\Delta\left(q_{4}, q_{1}, q_{2}, q_{0}\right)}{\Delta\left(q_{4}, q_{1}, q_{2}, q_{3}\right)} \otimes \frac{\Delta\left(q_{4}, q_{2}, q_{3}, q_{0}\right)}{\Delta\left(q_{4}, q_{1}, q_{3}, q_{0}\right)} \wedge \frac{\Delta\left(q_{4}, q_{1}, q_{3}, q_{0}\right)}{\Delta\left(q_{4}, q_{1}, q_{2}, q_{3}\right)} \\
& =0
\end{aligned}
$$

96 Lemma 11. The lower square of the diagram $E$ is commutative.

Proof. Let $\left(q_{0}, q_{1}, q_{2}, q_{3}, q_{4}\right) \in G_{5}(3)$ by applying map $d$ it becomes

$$
d\left(q_{0}, \ldots, q_{4}\right)=\sum_{i=0}^{4}(-1)^{i}\left(q_{0}, \ldots, \hat{q}_{i}, \ldots, q_{4}\right)
$$

apply map $f_{0}^{3}$, then

$$
f_{0}^{3} \circ d\left(q_{0}, \ldots, q_{4}\right)=\sum_{\substack{j \neq i \\ j=i+1}}^{4}(-1)^{j} \sum_{i=0}^{4}(-1)^{i} \Delta\left(q_{0}, \ldots, \hat{q}_{i}, \hat{q}_{j} \ldots, q_{4}\right) \otimes \frac{\Delta\left(q_{0}, \ldots, \hat{q}_{i+1}, \hat{q}_{j+1}, \ldots, q_{4}\right)}{\Delta\left(q_{0}, \ldots, \hat{q}_{i+2}, \hat{q}_{j+2}, \ldots, q_{4}\right)} \wedge
$$




$$
\frac{\triangle\left(q_{0}, \ldots, \hat{q}_{i+2}, \hat{q}_{j+2}, \ldots, q_{4}\right)}{\triangle\left(q_{0}, \ldots, \hat{q}_{i+3}, \hat{q}_{j+3}, \ldots, q_{4}\right)} \wedge \frac{\Delta\left(q_{0}, \ldots, \hat{q}_{i+3}, \hat{q}_{j+3}, \ldots, q_{4}\right)}{\triangle\left(q_{0}, \ldots, \hat{q}_{i+4}, \hat{q}_{j+4}, \ldots, q_{4}\right)}
$$

Applying morphism $f_{1}^{3}$ on $\left(q_{0}, \ldots, q_{4}\right) \in G_{5}(3)$. then

$$
\begin{aligned}
f_{1}^{3}\left(q_{0}, \ldots, q_{4}\right)= & -\frac{1}{3}\left(\sum _ { i = 0 } ^ { 4 } ( - 1 ) ^ { i } \left(\left\langle r\left(q_{i} \mid q_{0}, \ldots, \hat{q}_{i}, \ldots, q_{4}\right)\right\rangle_{2} \otimes \prod_{\substack{j \neq i \\
j=0}}^{4} \Delta\left(q_{0}, \ldots, \hat{q}_{i}, \hat{q}_{j}, \ldots, q_{4}\right)\right.\right. \\
& \left.-\prod_{\substack{j \neq i \\
j=0}}^{4} \Delta\left(q_{0}, \ldots, \hat{q}_{i}, \hat{q}_{j}, \ldots, q_{4}\right) \otimes\left[q_{i} \mid q_{0}, \ldots, \hat{q}_{i}, \ldots, q_{4}\right]_{2}\right)
\end{aligned}
$$

now apply map $\partial$

$$
\begin{aligned}
\partial \circ f_{1}^{3} & =-\frac{1}{3}\left(\sum _ { i = 0 } ^ { 4 } ( - 1 ) ^ { i } \left(r\left(q_{i} \mid q_{0}, \ldots, \hat{q}_{i}, \ldots, q_{4}\right) \otimes\left(1-r\left(q_{i} \mid q_{0}, \ldots, \hat{q}_{i}, \hat{q}_{j}, \ldots, q_{4}\right)\right) \wedge\right.\right. \\
& \prod_{\substack{j \neq i \\
j=0}}^{4} \Delta\left(q_{0}, \ldots, \hat{q}_{i}, \hat{q}_{j}, \ldots, q_{4}\right)-\left(1-r\left(q_{i} \mid q_{0}, \ldots, \hat{q}_{i}, \hat{q}_{j}, \ldots, q_{4}\right)\right) \otimes r\left(q_{i} \mid q_{0}, \ldots, \hat{q}_{i}, \hat{q}_{j}, \ldots, q_{4}\right) \wedge \\
& \prod_{\substack{j \neq i \\
j=0}}^{4} \Delta\left(q_{0}, \ldots, \hat{q}_{i}, \hat{q}_{j}, \ldots, q_{4}\right)-\prod_{\substack{j \neq i \\
j=0}}^{4} \Delta\left(q_{0}, \ldots, \hat{q}_{i}, \hat{q}_{j}, \ldots, q_{4}\right) \otimes\left(1-r\left(q_{i} \mid q_{0}, \ldots, \hat{q}_{i}, \ldots, q_{4}\right)\right) \wedge \\
& \left.r\left(q_{i} \mid q_{0}, \ldots, \hat{q}_{i}, \ldots, q_{4}\right)\right)
\end{aligned}
$$

after using tensor, wedge and Siegel cross ratio properties [15], it becomes

$$
\begin{aligned}
\partial \circ f_{1}^{3}\left(q_{0}, \ldots, q_{4}\right)= & \sum_{\substack{j \neq i \\
j=i+1}}^{4}(-1)^{j} \sum_{i=0}^{4}(-1)^{i} \Delta\left(q_{0}, \ldots, \hat{q}_{i}, \hat{q}_{j} \ldots, q_{4}\right) \otimes \frac{\Delta\left(q_{0}, \ldots, \hat{q}_{i+1}, \hat{q}_{j+1}, \ldots, q_{4}\right)}{\Delta\left(q_{0}, \ldots, \hat{q}_{i+2}, \hat{q}_{j+2}, \ldots, q_{4}\right)} \wedge \\
& \frac{\Delta\left(q_{0}, \ldots, \hat{q}_{i+2}, \hat{q}_{j+2}, \ldots, q_{4}\right)}{\Delta\left(q_{0}, \ldots, \hat{q}_{i+3}, \hat{q}_{j+3}, \ldots, q_{4}\right)} \wedge \frac{\Delta\left(q_{0}, \ldots, \hat{q}_{i+3}, \hat{q}_{j+3}, \ldots, q_{4}\right)}{\Delta\left(q_{0}, \ldots, \hat{q}_{i+4}, \hat{q}_{j+4}, \ldots, q_{4}\right)}
\end{aligned}
$$

${ }_{97}$ from Eq.(26) and Eq.(29) it is observed that, $f_{0}^{3} \circ d=\partial \circ f_{1}^{3}$

98 3.3. Weight $n=4$

In this weight connect the sub-complexes of Cathelineau's infinitesimal and Grassmannian

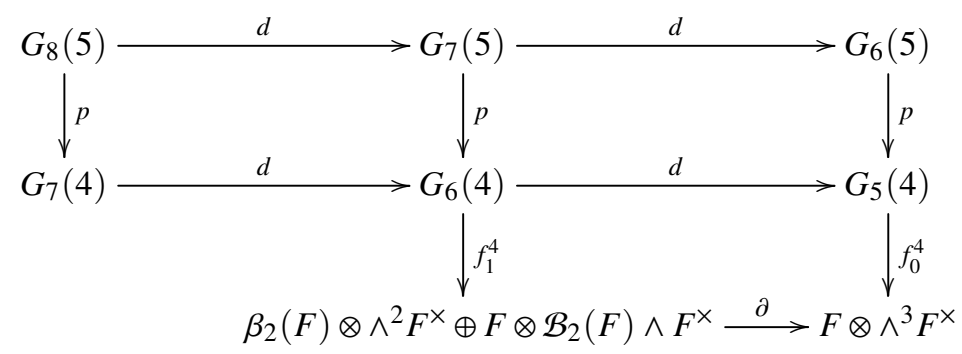

where

$$
f_{0}^{4}\left(q_{0}, \ldots, q_{4}\right)=\sum_{i=0}^{4}(-1)^{i} \Delta\left(q_{0}, \ldots, \hat{q}_{i}, \ldots, q_{4}\right) \otimes \frac{\Delta\left(q_{0}, \ldots, \hat{q}_{i+1}, \ldots, q_{4}\right)}{\Delta\left(q_{0}, \ldots, \hat{q}_{i+2}, \ldots, q_{4}\right)} \wedge \frac{\Delta\left(q_{0}, \ldots, \hat{q}_{i+2}, \ldots, q_{4}\right)}{\Delta\left(q_{0}, \ldots, \hat{q}_{i+3}, \ldots, q_{4}\right)} \wedge
$$




$$
\frac{\triangle\left(q_{0}, \ldots, \hat{q}_{i+3}, \ldots, q_{4}\right)}{\triangle\left(q_{0}, \ldots, \hat{q}_{i+4}, \ldots, q_{4}\right)} \quad(\bmod 5)
$$

and

$$
\begin{aligned}
f_{1}^{4}\left(q_{0}, \ldots, q_{5}\right) & =\frac{1}{6}\left(\sum _ { \substack { i \neq j \\
i = 0 \\
j = i + 1 } } ^ { 5 } ( - 1 ) ^ { i } \left(\left\langle r\left(q_{i}, q_{j} \mid q_{0}, \ldots, \hat{q}_{i}, \hat{q}_{j}, \ldots, q_{5}\right)\right\rangle_{2} \otimes \prod_{\substack{k \neq i \\
k=i+1}}^{5} \Delta\left(q_{0}, \ldots, \hat{q}_{i}, \hat{q}_{k}, \ldots, q_{5}\right) \wedge\right.\right. \\
& \prod_{\substack{k \neq j \\
k=j+1}}^{5} \Delta\left(q_{0}, \ldots, \hat{q}_{j}, \hat{q}_{k}, \ldots, q_{5}\right)-\prod_{\substack{k \neq i \\
k=i+1}}^{5} \Delta\left(q_{0}, \ldots, \hat{q}_{i}, \hat{q}_{k}, \ldots, q_{5}\right) \otimes\left[r\left(q_{i}, q_{j} \mid q_{0}, \ldots, \hat{q}_{i}, \hat{q}_{j}, \ldots, q_{5}\right)\right]_{2} \otimes \\
& \prod_{\substack{k \neq j \\
k=j+1}}^{5} \Delta\left(q_{0}, \ldots, \hat{q}_{j}, \hat{q}_{k}, \ldots, q_{5}\right)+\prod_{\substack{k \neq j \\
k=j+1}}^{5} \Delta\left(q_{0}, \ldots, \hat{q}_{j}, \hat{q}_{k}, \ldots, q_{5}\right) \otimes\left[r\left(q_{i}, q_{j} \mid q_{0}, \ldots, \hat{q}_{i}, \hat{q}_{j}, \ldots, q_{5}\right)\right]_{2} \otimes \\
& \left.\prod_{\substack{k \neq i \\
k=i+1}}^{5} \Delta\left(q_{0}, \ldots, \hat{q}_{i}, \hat{q}_{k}, \ldots, q_{5}\right)\right)
\end{aligned}
$$

99 Lemma 12. $f_{0}^{4} \circ d=\partial \circ f_{1}^{4}$.

Proof. Let the five points be $\left(q_{0}, q_{1}, q_{2}, q_{3}, q_{4}, q_{5}\right) \in G_{6}(4)$, now apply map $d$, then

$$
d\left(q_{0}, \ldots, q_{5}\right)=\sum_{i=0}^{5}(-1)^{i}\left(q_{0}, \ldots, \hat{q}_{i}, \ldots, q_{5}\right)
$$

now apply morphism $f_{0}^{4}$

$$
\begin{aligned}
f_{0}^{4} \circ d\left(q_{0}, \ldots, q_{5}\right)= & \sum_{\substack{k \neq i \\
k=i+1}}^{5}(-1)^{k} \sum_{i=0}^{5}(-1)^{i} \Delta\left(q_{0}, \ldots, \hat{q}_{i}, \hat{q}_{k} \ldots, q_{5}\right) \otimes \frac{\Delta\left(q_{0}, \ldots, \hat{q}_{i+1}, \hat{q}_{k+1}, \ldots, q_{5}\right)}{\triangle\left(q_{0}, \ldots, \hat{q}_{i+2}, \hat{q}_{k+2}, \ldots, q_{5}\right)} \wedge \\
& \frac{\Delta\left(q_{0}, \ldots, \hat{q}_{i+2}, \hat{q}_{k+2}, \ldots, q_{5}\right)}{\Delta\left(q_{0}, \ldots, \hat{q}_{i+3}, \hat{q}_{k+3}, \ldots, q_{5}\right)} \wedge \frac{\Delta\left(q_{0}, \ldots, \hat{q}_{i+3}, \hat{q}_{k+3}, \ldots, q_{5}\right)}{\Delta\left(q_{0}, \ldots, \hat{q}_{i+4}, \hat{q}_{k+4}, \ldots, q_{5}\right)} \wedge \frac{\Delta\left(q_{0}, \ldots, \hat{q}_{i+4}, \hat{q}_{k+4}, \ldots, q_{5}\right)}{\Delta\left(q_{0}, \ldots, \hat{q}_{i+5}, \hat{q}_{k+5}, \ldots, q_{5}\right)}
\end{aligned}
$$

Apply map $f_{1}^{4}$ on $\left(q_{0}, \ldots, q_{5}\right) \in G_{6}(4)$, then

$$
\begin{aligned}
& f_{1}^{4}\left(q_{0}, \ldots, q_{5}\right)=\frac{1}{6}\left(\sum _ { \substack { i \neq j \\
i = 0 \\
j = i + 1 } } ^ { 5 } ( - 1 ) ^ { i } \left(\langle r ( q _ { i } , q _ { j } | q _ { 0 } , \ldots , \hat { q } _ { i } , \hat { q } _ { j } , \ldots , q _ { 5 } ) \rangle _ { 2 } \otimes \prod _ { \substack { k \neq i \\
k = i + 1 } } ^ { 5 } \Delta ( q _ { 0 } , \ldots , \hat { q } _ { i } , \hat { q } _ { k } , \ldots , q _ { 5 } ) \wedge \prod _ { \substack { k \neq j \\
k = j + 1 } } ^ { 5 } \Delta \left(q_{0}, \ldots,\right.\right.\right. \\
& \left.\hat{q}_{j}, \hat{q}_{k}, \ldots, q_{5}\right)-\prod_{\substack{k \neq i \\
k=i+1}}^{5} \Delta\left(q_{0}, \ldots, \hat{q}_{i}, \hat{q}_{k}, \ldots, q_{5}\right) \otimes\left[r\left(q_{i}, q_{j} \mid q_{0}, \ldots, \hat{q}_{i}, \hat{q}_{j}, \ldots, q_{5}\right)\right]_{2} \otimes \prod_{\substack{k \neq j \\
k=j+1}}^{5} \Delta\left(q_{0}, \ldots, \hat{q}_{j}, \hat{q}_{k}, \ldots, q_{5}\right) \\
& \left.+\prod_{\substack{k \neq j \\
k=j+1}}^{5} \Delta\left(q_{0}, \ldots, \hat{q}_{j}, \hat{q}_{k}, \ldots, q_{5}\right) \otimes\left[r\left(q_{i}, q_{j} \mid q_{0}, \ldots, \hat{q}_{i}, \hat{q}_{j}, \ldots, q_{5}\right)\right]_{2} \otimes \prod_{\substack{k \neq i \\
k=i+1}}^{5} \Delta\left(q_{0}, \ldots, \hat{q}_{i}, \hat{q}_{k}, \ldots, q_{5}\right)\right)
\end{aligned}
$$


On applying map $\partial$

$$
\begin{aligned}
& \partial \circ f_{1}^{4}\left(q_{0}, \ldots, q_{5}\right)=\frac{1}{6}\left(\sum _ { \substack { i \neq j \\
i = 0 \\
j = i + 1 } } ^ { 5 } ( - 1 ) ^ { i } \left[\left(r\left(q_{i}, q_{j} \mid q_{0}, \ldots, \hat{q}_{i}, \hat{q}_{j}, \ldots, q_{5}\right)\right) \otimes\left(r\left(q_{i}, q_{j} \mid q_{0}, \ldots, \hat{q}_{i}, \hat{q}_{j}, \ldots, q_{5}\right)-1\right) \wedge\right.\right. \\
& \prod_{\substack{k \neq i \\
k=i+1}}^{5} \Delta\left(q_{0}, \ldots, \hat{q}_{i}, \hat{q}_{k}, \ldots, q_{5}\right) \wedge \prod_{\substack{k \neq j \\
k=0}}^{5} \Delta\left(q_{0}, \ldots, \hat{q}_{j}, \hat{q}_{k}, \ldots, q_{5}\right)-\left(1-r\left(q_{i}, q_{j} \mid q_{0}, \ldots, \hat{q}_{i}, \hat{q}_{j}, \ldots, q_{5}\right)\right) \otimes \\
& \left(r\left(q_{i}, q_{j} \mid q_{0}, \ldots, \hat{q}_{i}, \hat{q}_{j}, \ldots, q_{5}\right)\right) \wedge \prod_{\substack{k \neq i \\
k=i+1}}^{5} \Delta\left(q_{0}, \ldots, \hat{q}_{i}, \hat{q}_{k}, \ldots, q_{5}\right) \wedge \prod_{\substack{k \neq j \\
k=j+1}}^{5} \Delta\left(q_{0}, \ldots, \hat{q}_{j}, \hat{q}_{k}, \ldots, q_{5}\right)- \\
& \prod_{\substack{k \neq i \\
k=i+1}}^{5} \Delta\left(q_{0}, \ldots, \hat{q}_{i}, \hat{q}_{k}, \ldots, q_{5}\right) \otimes\left(1-r\left(q_{i}, q_{j} \mid q_{0}, \ldots, \hat{q}_{i}, \hat{q}_{j}, \ldots, q_{5}\right)\right) \wedge r\left(q_{i}, q_{j} \mid q_{0}, \ldots, \hat{q}_{i}, \hat{q}_{j}, \ldots, q_{5}\right) \wedge \\
& \prod_{\substack{k \neq j \\
k=j+1}}^{5} \Delta\left(q_{0}, \ldots, \hat{q}_{j}, \hat{q}_{k}, \ldots, q_{5}\right)+\prod_{\substack{k \neq j \\
k=0}}^{5} \Delta\left(q_{0}, \ldots, \hat{q}_{j}, \hat{q}_{k}, \ldots, q_{5}\right) \otimes\left(1-r\left(q_{i}, q_{j} \mid q_{0}, \ldots, \hat{q}_{i}, \hat{q}_{j}, \ldots, q_{5}\right)\right) \wedge \\
& \left.\left.r\left(q_{i}, q_{j} \mid q_{0}, \ldots, \hat{q}_{i}, \hat{q}_{j}, \ldots, q_{5}\right) \wedge \prod_{\substack{k \neq i \\
k=i+1}}^{5} \Delta\left(q_{0}, \ldots, \hat{q}_{i}, \hat{q}_{k}, \ldots, q_{5}\right)\right]\right)
\end{aligned}
$$

after using tensor,wedge, Siegel cross ratio properties and dummy indices it becomes

$$
\begin{aligned}
\partial \circ f_{1}^{4}\left(q_{0}, \ldots, q_{5}\right)= & \sum_{\substack{k \neq i \\
k=i+1}}^{5}(-1)^{k} \sum_{i=0}^{5}(-1)^{i} \Delta\left(q_{0}, \ldots, \hat{q}_{i}, \hat{q}_{k} \ldots, q_{5}\right) \otimes \frac{\Delta\left(q_{0}, \ldots, \hat{q}_{i+1}, \hat{q}_{k+1}, \ldots, q_{5}\right)}{\Delta\left(q_{0}, \ldots, \hat{q}_{i+2}, \hat{q}_{k+2}, \ldots, q_{5}\right)} \wedge \\
& \frac{\Delta\left(q_{0}, \ldots, \hat{q}_{i+2}, \hat{q}_{k+2}, \ldots, q_{5}\right)}{\Delta\left(q_{0}, \ldots, \hat{q}_{i+3}, \hat{q}_{k+3}, \ldots, q_{5}\right)} \wedge \frac{\Delta\left(q_{0}, \ldots, \hat{q}_{i+3}, \hat{q}_{k+3}, \ldots, q_{5}\right)}{\Delta\left(q_{0}, \ldots, \hat{q}_{i+4}, \hat{q}_{k+4}, \ldots, q_{5}\right)} \wedge \frac{\Delta\left(q_{0}, \ldots, \hat{q}_{i+4}, \hat{q}_{k+4}, \ldots, q_{5}\right)}{\Delta\left(q_{0}, \ldots, \hat{q}_{i+5}, \hat{q}_{k+5}, \ldots, q_{5}\right)}
\end{aligned}
$$

Eq.(33) and Eq.(36) proves $f_{0}^{4} \circ d=\partial \circ f_{1}^{4} \quad \square$

3.4. Weight $n=5$

Connect the two sub-complexes given as

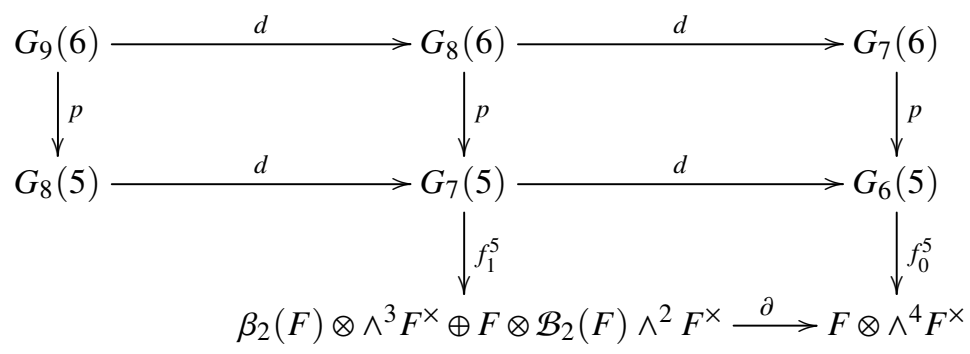

where

$$
\begin{array}{r}
f_{0}^{5}\left(q_{0}, \ldots, q_{5}\right)=\sum_{i=0}^{5}(-1)^{i} \Delta\left(q_{0}, \ldots, \hat{q}_{i}, \ldots, q_{5}\right) \otimes \frac{\Delta\left(q_{0}, \ldots, \hat{q}_{i+1}, \ldots, q_{5}\right)}{\Delta\left(q_{0}, \ldots, \hat{q}_{i+2}, \ldots, q_{5}\right)} \wedge \frac{\Delta\left(q_{0}, \ldots, \hat{q}_{i+2}, \ldots, q_{5}\right)}{\Delta\left(q_{0}, \ldots, \hat{q}_{i+3}, \ldots, q_{5}\right)} \\
\frac{\Delta\left(q_{0}, \ldots, \hat{q}_{i+3}, \ldots, q_{5}\right)}{\Delta\left(q_{0}, \ldots, \hat{q}_{i+4}, \ldots, q_{5}\right)} \wedge \frac{\Delta\left(q_{0}, \ldots, \hat{q}_{i+4}, \ldots, q_{5}\right)}{\Delta\left(q_{0}, \ldots, \hat{q}_{i+5}, \ldots, q_{5}\right)} \quad(\bmod 6)
\end{array}
$$


and

$$
\begin{aligned}
& f_{1}^{5}\left(q_{0}, \ldots, q_{6}\right)=\frac{1}{10}\left(\sum _ { \substack { i \neq j \neq k \\
i = 0 \\
j = i + 1 \\
k = i + 2 } } ^ { 6 } ( - 1 ) ^ { i } \left(\left\langle r\left(q_{i}, q_{j}, q_{k} \mid q_{0}, \ldots, \hat{q}_{i}, \hat{q}_{j}, \hat{q}_{k}, \ldots, q_{6}\right)\right\rangle_{2} \otimes \prod_{\substack{l \neq i \\
l=i+1}}^{6} \Delta\left(q_{0}, \ldots, \hat{q}_{i}, \hat{q}_{l}, \ldots, q_{6}\right) \wedge\right.\right. \\
& \prod_{\substack{l \neq j \\
l=j+1}}^{6} \Delta\left(q_{0}, \ldots, \hat{q}_{j}, \hat{q}_{l}, \ldots, q_{6}\right) \wedge \prod_{\substack{l \neq k \\
l=k+1}}^{6} \Delta\left(q_{0}, \ldots, \hat{q}_{k}, \hat{q}_{l}, \ldots, q_{6}\right)-\prod_{\substack{l \neq i \\
l=i+1}}^{6} \Delta\left(q_{0}, \ldots, \hat{q}_{i}, \hat{q}_{l}, \ldots, q_{6}\right) \otimes \\
& {\left[r\left(q_{i}, q_{j}, q_{k} \mid q_{0}, \ldots, \hat{q}_{i}, \hat{q}_{j}, \hat{q}_{k}, \ldots, q_{6}\right)\right]_{2} \otimes \prod_{\substack{l \neq j \\
l=j+1}}^{6} \Delta\left(q_{0}, \ldots, \hat{q}_{j}, \hat{q}_{l}, \ldots, q_{6}\right) \wedge \prod_{\substack{l \neq k \\
l=k+1}}^{6} \Delta\left(q_{0}, \ldots, \hat{q}_{k}, \hat{q}_{l}, \ldots, q_{6}\right)+} \\
& \prod_{\substack{l \neq j \\
l=j+1}}^{6} \Delta\left(q_{0}, \ldots, \hat{q}_{j}, \hat{q}_{l}, \ldots, q_{6}\right) \otimes\left[r\left(q_{i}, q_{j}, q_{k} \mid q_{0}, \ldots, \hat{q}_{i}, \hat{q}_{j}, \hat{q}_{k}, \ldots, q_{6}\right)\right]_{2} \otimes \prod_{\substack{l \neq i \\
l=i+1}}^{6} \Delta\left(q_{0}, \ldots, \hat{q}_{i}, \hat{q}_{l}, \ldots, q_{6}\right) \\
& \wedge \prod_{\substack{l \neq k \\
l=k+1}}^{6} \Delta\left(q_{0}, \ldots, \hat{q}_{k}, \hat{q}_{l}, \ldots, q_{6}\right)-\prod_{\substack{l \neq k \\
l=k+1}}^{6} \Delta\left(q_{0}, \ldots, \hat{q}_{k}, \hat{q}_{l}, \ldots, q_{6}\right) \otimes\left[r\left(q_{i}, q_{j}, q_{k} \mid q_{0}, \ldots, \hat{q}_{i}, \hat{q}_{j}, \hat{q}_{k}, \ldots, q_{6}\right)\right]_{2} \\
& \left.\otimes \prod_{\substack{l \neq i \\
l=i+1}}^{6} \Delta\left(q_{0}, \ldots, \hat{q}_{i}, \hat{q}_{l}, \ldots, q_{6}\right) \wedge \prod_{\substack{l \neq j \\
l=j+1}}^{6} \Delta\left(q_{0}, \ldots, \hat{q}_{j}, \hat{q}_{l}, \ldots, q_{6}\right)\right)(\bmod 7)
\end{aligned}
$$

Lemma 13. $f_{0}^{5} \circ d=\partial \circ f_{1}^{5}$.

Proof. Let $\left(q_{0}, \ldots, q_{6}\right) \in G_{7}(5)$ on apply map $d$

$$
d\left(q_{0}, \ldots, q_{6}\right)=\sum_{i=0}^{6}(-1)^{i}\left(q_{0}, \ldots, \hat{q}_{i}, \ldots, q_{6}\right)
$$

By applying morphism $f_{0}^{5}$

$$
\begin{aligned}
& f_{0}^{5} \circ d\left(q_{0}, \ldots, q_{6}\right)=\sum_{\substack{l \neq i \\
l=i+1}}^{6}(-1)^{l} \sum_{i=0}^{6}(-1)^{i} \Delta\left(q_{0}, \ldots, \hat{q}_{i}, \hat{q}_{l} \ldots, q_{6}\right) \otimes \frac{\Delta\left(q_{0}, \ldots, \hat{q}_{i+1}, \hat{q}_{l+1}, \ldots, q_{6}\right)}{\Delta\left(q_{0}, \ldots, \hat{q}_{i+2}, \hat{q}_{j l+2}, \ldots, q_{6}\right)} \wedge \\
& \frac{\Delta\left(q_{0}, \ldots, \hat{q}_{i+2}, \hat{q}_{l+2}, \ldots, q_{6}\right)}{\Delta\left(q_{0}, \ldots, \hat{q}_{i+3}, \hat{q}_{l+3}, \ldots, q_{6}\right)} \wedge \frac{\Delta\left(q_{0}, \ldots, \hat{q}_{i+3}, \hat{q}_{l+3}, \ldots, q_{6}\right)}{\Delta\left(q_{0}, \ldots, \hat{q}_{i+4}, \hat{q}_{l+4}, \ldots, q_{6}\right)} \wedge \\
& \frac{\Delta\left(q_{0}, \ldots, \hat{q}_{i+4}, \hat{q}_{l+4}, \ldots, q_{6}\right)}{\Delta\left(q_{0}, \ldots, \hat{q}_{i+5}, \hat{q}_{l+5}, \ldots, q_{6}\right)} \wedge \frac{\Delta\left(q_{0}, \ldots, \hat{q}_{i+5}, \hat{q}_{l+5}, \ldots, q_{6}\right)}{\Delta\left(q_{0}, \ldots, \hat{q}_{i+6}, \hat{q}_{l+6}, \ldots, q_{6}\right)}
\end{aligned}
$$

Apply $f_{1}^{5}$ on $\left(q_{0}, \ldots, q_{6}\right) \in G_{7}(5)$

$$
\begin{aligned}
f_{1}^{5}\left(q_{0}, \ldots, q_{6}\right)= & \frac{1}{10}\left(\sum _ { \substack { i \neq j \neq k \\
i = 0 \\
j = i + 1 \\
k = i + 2 } } ^ { 6 } ( - 1 ) ^ { i } \left(\left\langle r\left(q_{i}, q_{j}, q_{k} \mid q_{0}, \ldots, \hat{q}_{i}, \hat{q}_{j}, \hat{q}_{k}, \ldots, q_{6}\right)\right\rangle_{2} \otimes \prod_{\substack{l \neq i \\
l=i+1}}^{6} \Delta\left(q_{0}, \ldots, \hat{q}_{i}, \hat{q}_{l}, \ldots, q_{6}\right) \wedge\right.\right. \\
& \prod_{\substack{l \neq j \\
l=j+1}}^{6} \Delta\left(q_{0}, \ldots, \hat{q}_{j}, \hat{q}_{l}, \ldots, q_{6}\right) \wedge \prod_{\substack{l \neq k \\
l=k+1}}^{6} \Delta\left(q_{0}, \ldots, \hat{q}_{k}, \hat{q}_{l}, \ldots, q_{6}\right)-\prod_{\substack{l \neq i \\
l=i+1}}^{6} \Delta\left(q_{0}, \ldots, \hat{q}_{i}, \hat{q}_{l}, \ldots, q_{6}\right) \otimes
\end{aligned}
$$




$$
\begin{aligned}
& {\left[r\left(q_{i}, q_{j}, q_{k} \mid q_{0}, \ldots, \hat{q}_{i}, \hat{q}_{j}, \hat{q}_{k}, \ldots, q_{6}\right)\right]_{2} \otimes \prod_{\substack{l \neq j \\
l=j+1}}^{6} \Delta\left(q_{0}, \ldots, \hat{q}_{j}, \hat{q}_{l}, \ldots, q_{6}\right) \wedge \prod_{\substack{l \neq k \\
l=k+1}}^{6} \Delta\left(q_{0}, \ldots, \hat{q}_{k}, \hat{q}_{l}, \ldots, q_{6}\right)} \\
& +\prod_{\substack{l \neq j \\
l=j+1}}^{6} \Delta\left(q_{0}, \ldots, \hat{q}_{j}, \hat{q}_{l}, \ldots, q_{6}\right) \otimes\left[r\left(q_{i}, q_{j}, q_{k} \mid q_{0}, \ldots, \hat{q}_{i}, \hat{q}_{j}, \hat{q}_{k}, \ldots, q_{6}\right)\right]_{2} \otimes \prod_{\substack{l \neq i \\
l=i+1}}^{6} \Delta\left(q_{0}, \ldots, \hat{q}_{i}, \hat{q}_{l}, \ldots, q_{6}\right) \wedge \\
& \prod_{\substack{l \neq k \\
l=k+1}}^{6} \Delta\left(q_{0}, \ldots, \hat{q}_{k}, \hat{q}_{l}, \ldots, q_{6}\right)-\prod_{\substack{l \neq k \\
l=k+1}}^{6} \Delta\left(q_{0}, \ldots, \hat{q}_{k}, \hat{q}_{l}, \ldots, q_{6}\right) \otimes\left[r\left(q_{i}, q_{j}, q_{k} \mid q_{0}, \ldots, \hat{q}_{i}, \hat{q}_{j}, \hat{q}_{k}, \ldots, q_{6}\right)\right]_{2} \otimes \\
& \left.\prod_{\substack{l \neq i \\
l=i+1}}^{6} \Delta\left(q_{0}, \ldots, \hat{q}_{i}, \hat{q}_{l}, \ldots, q_{6}\right) \wedge \prod_{\substack{l \neq j \\
l=j+1}}^{6} \Delta\left(q_{0}, \ldots, \hat{q}_{j}, \hat{q}_{l}, \ldots, q_{6}\right)\right)
\end{aligned}
$$

Apply map $\partial$

$$
\begin{aligned}
& \partial \circ f_{1}^{5}\left(q_{0}, \ldots, q_{6}\right)=\frac{1}{10}\left(\sum _ { \substack { i \neq j \neq k \\
i = 0 } } ^ { 6 } ( - 1 ) ^ { i } \left(r ( q _ { i } , q _ { j } , q _ { k } | q _ { 0 } , \ldots , \hat { q } _ { i } , \hat { q } _ { j } , \hat { q } _ { k } , \ldots , q _ { 6 } ) \otimes \left(1-r\left(q_{i}, q_{j}, q_{k} \mid q_{0}, \ldots, \hat{q}_{i}, \hat{q}_{j}, \hat{q}_{k},\right.\right.\right.\right. \\
& \begin{array}{l}
i=0 \\
j=i+1 \\
k=i+2
\end{array} \\
& \left.\left.\ldots, q_{6}\right)\right) \wedge \prod_{\substack{l \neq i \\
l=0 i+1}}^{6} \Delta\left(q_{0}, \ldots, \hat{q}_{i}, \hat{q}_{l}, \ldots, q_{6}\right) \wedge \prod_{\substack{l \neq j \\
l=j+1}}^{6} \Delta\left(q_{0}, \ldots, \hat{q}_{j}, \hat{q}_{l}, \ldots, q_{6}\right) \wedge \prod_{\substack{l \neq k \\
l=k+1}}^{6} \Delta\left(q_{0}, \ldots, \hat{q}_{k}, \hat{q}_{l}, \ldots, q_{6}\right) \\
& -\left(1-r\left(q_{i}, q_{j}, q_{k} \mid q_{0}, \ldots, \hat{q}_{i}, \hat{q}_{j}, \hat{q}_{k}, \ldots, q_{6}\right)\right) \otimes\left(r\left(q_{i}, q_{j}, q_{k} \mid q_{0}, \ldots, \hat{q}_{i}, \hat{q}_{j}, \hat{q}_{k}, \ldots, q_{6}\right)\right) \wedge \\
& \prod_{\substack{l \neq i \\
l=i+1}}^{6} \Delta\left(q_{0}, \ldots, \hat{q}_{i}, \hat{q}_{l}, \ldots, q_{6}\right) \wedge \prod_{\substack{l \neq j \\
l=j+1}}^{6} \Delta\left(q_{0}, \ldots, \hat{q}_{j}, \hat{q}_{l}, \ldots, q_{6}\right) \wedge \prod_{\substack{l \neq k \\
l=k+1}}^{6} \Delta\left(q_{0}, \ldots, \hat{q}_{k}, \hat{q}_{l}, \ldots, q_{6}\right) \\
& -\prod_{\substack{l \neq i \\
l=i+1}}^{6} \Delta\left(q_{0}, \ldots, \hat{q}_{i}, \hat{q}_{l}, \ldots, q_{6}\right) \otimes\left[r\left(q_{i}, q_{j}, q_{k} \mid q_{0}, \ldots, \hat{q}_{i}, \hat{q}_{j}, \hat{q}_{k}, \ldots, q_{6}\right)\right]_{2} \otimes \prod_{\substack{l \neq j \\
l=j+1}}^{6} \Delta\left(q_{0}, \ldots, \hat{q}_{j}, \hat{q}_{l}, \ldots, q_{6}\right) \wedge \\
& \prod_{\substack{l \neq k \\
l=k+1}}^{6} \Delta\left(q_{0}, \ldots, \hat{q}_{k}, \hat{q}_{l}, \ldots, q_{6}\right)+\prod_{\substack{l \neq j \\
l=j+1}}^{6} \Delta\left(q_{0}, \ldots, \hat{q}_{j}, \hat{q}_{l}, \ldots, q_{6}\right) \otimes\left[r\left(q_{i}, q_{j}, q_{k} \mid q_{0}, \ldots, \hat{q}_{i}, \hat{q}_{j}, \hat{q}_{k}, \ldots, q_{6}\right)\right]_{2} \otimes \\
& \prod_{\substack{l \neq i \\
l=i+1}}^{6} \Delta\left(q_{0}, \ldots, \hat{q}_{i}, \hat{q}_{l}, \ldots, q_{6}\right) \wedge \prod_{\substack{l \neq k \\
l=k+1}}^{6} \Delta\left(q_{0}, \ldots, \hat{q}_{k}, \hat{q}_{l}, \ldots, q_{6}\right)-\prod_{\substack{l \neq k \\
l=k+1}}^{6} \Delta\left(q_{0}, \ldots, \hat{q}_{k}, \hat{q}_{l}, \ldots, q_{6}\right) \otimes \\
& \left.\left[r\left(q_{i}, q_{j}, q_{k} \mid q_{0}, \ldots, \hat{q}_{i}, \hat{q}_{j}, \hat{q}_{k}, \ldots, q_{6}\right)\right]_{2} \otimes \prod_{\substack{l \neq i \\
l=i+1}}^{6} \Delta\left(q_{0}, \ldots, \hat{q}_{i}, \hat{q}_{l}, \ldots, q_{6}\right) \wedge \prod_{\substack{l \neq j \\
l=j+1}}^{6} \Delta\left(q_{0}, \ldots, \hat{q}_{j}, \hat{q}_{l}, \ldots, q_{6}\right)\right)
\end{aligned}
$$

after using tensor,wedge, Siegel cross ratio properties and dummy indices it becomes

$$
\begin{array}{r}
\partial \circ f_{1}^{5}\left(q_{0}, \ldots, q_{6}\right)=\sum_{\substack{l \neq i \\
l=i+1}}^{6}(-1)^{l} \sum_{i=0}^{6}(-1)^{i} \Delta\left(q_{0}, \ldots, \hat{q}_{i}, \hat{q}_{l} \ldots, q_{6}\right) \otimes \frac{\Delta\left(q_{0}, \ldots, \hat{q}_{i+1}, \hat{q}_{l+1}, \ldots, q_{6}\right)}{\Delta\left(q_{0}, \ldots, \hat{q}_{i+2}, \hat{q}_{j l+2}, \ldots, q_{6}\right)} \wedge \\
\frac{\Delta\left(q_{0}, \ldots, \hat{q}_{i+2}, \hat{q}_{l+2}, \ldots, q_{6}\right)}{\Delta\left(q_{0}, \ldots, \hat{q}_{i+3}, \hat{q}_{l+3}, \ldots, q_{6}\right)} \wedge \frac{\Delta\left(q_{0}, \ldots, \hat{q}_{i+3}, \hat{q}_{l+3}, \ldots, q_{6}\right)}{\Delta\left(q_{0}, \ldots, \hat{q}_{i+4}, \hat{q}_{l+4}, \ldots, q_{6}\right)} \wedge
\end{array}
$$




$$
\frac{\Delta\left(q_{0}, \ldots, \hat{q}_{i+4}, \hat{q}_{l+4}, \ldots, q_{6}\right)}{\triangle\left(q_{0}, \ldots, \hat{q}_{i+5}, \hat{q}_{l+5}, \ldots, q_{6}\right)} \wedge \frac{\Delta\left(q_{0}, \ldots, \hat{q}_{i+5}, \hat{q}_{l+5}, \ldots, q_{6}\right)}{\Delta\left(q_{0}, \ldots, \hat{q}_{i+6}, \hat{q}_{l+6}, \ldots, q_{6}\right)}
$$

Hence Eq.(40) and Eq.(43) proves $f_{0}^{5} \circ d=\partial \circ f_{1}^{5}$

3.5. Weight $n=6$

Connect the two simplicial complexes Grassmannian and Cathelineau as

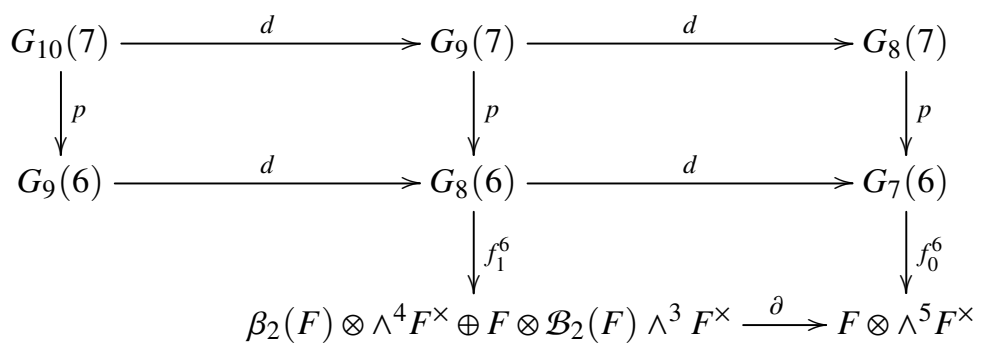

where

$$
\begin{aligned}
f_{0}^{6}\left(q_{0}, \ldots, q_{6}\right)= & \sum_{i=0}^{6}(-1)^{i} \triangle\left(q_{0}, \ldots, \hat{q}_{i}, \ldots, q_{6}\right) \otimes \frac{\Delta\left(q_{0}, \ldots, \hat{q}_{i+1}, \ldots, q_{6}\right)}{\Delta\left(q_{0}, \ldots, \hat{q}_{i+2}, \ldots, q_{6}\right)} \wedge \frac{\Delta\left(q_{0}, \ldots, \hat{q}_{i+2}, \ldots, q_{6}\right)}{\Delta\left(q_{0}, \ldots, \hat{q}_{i+3}, \ldots, q_{6}\right)} \\
& \frac{\Delta\left(q_{0}, \ldots, \hat{q}_{i+3}, \ldots, q_{6}\right)}{\Delta\left(q_{0}, \ldots, \hat{q}_{i+4}, \ldots, q_{6}\right)} \wedge \frac{\Delta\left(q_{0}, \ldots, \hat{q}_{i+4}, \ldots, q_{6}\right)}{\Delta\left(q_{0}, \ldots, \hat{q}_{i+5}, \ldots, q_{6}\right)} \wedge \frac{\Delta\left(q_{0}, \ldots, \hat{q}_{i+5}, \ldots, q_{6}\right)}{\Delta\left(q_{0}, \ldots, \hat{q}_{i+6}, \ldots, q_{6}\right)} \quad(\bmod 7)
\end{aligned}
$$

and

$$
\begin{aligned}
& f_{1}^{6}\left(q_{0}, \ldots, q_{7}\right)=-\frac{1}{15}\left(\sum _ { \substack { i _ { 0 } = 0 \\
i _ { 1 } = i _ { 0 } + 1 \\
i _ { 2 } = i _ { 0 } + 2 \\
i _ { 3 } = i _ { 0 } + 1 } } ^ { 7 } ( - 1 ) ^ { i _ { 0 } } \left(\left\langle r\left(q_{i_{0}}, q_{i_{1}}, q_{i_{2}}, q_{i_{3}} \mid q_{0}, \ldots, \hat{q}_{i_{0}}, \hat{q}_{i_{1}}, \hat{q}_{i_{2}}, \hat{q}_{i_{3}}, \ldots, q_{7}\right)\right\rangle_{2} \otimes\right.\right. \\
& \prod_{\substack{j \neq i_{0} \\
j=i_{0}+1}}^{7} \Delta\left(q_{0}, \ldots, \hat{q}_{i_{0}}, \hat{q}_{j}, \ldots, q_{7}\right) \wedge \prod_{\substack{j \neq i_{1} \\
j=i_{1}+1}}^{7} \Delta\left(q_{0}, \ldots, \hat{q}_{i_{1}}, \hat{q}_{j}, \ldots, q_{7}\right) \wedge \prod_{\substack{j \neq i_{2} \\
j=i_{2}+1}}^{7} \Delta\left(q_{0}, \ldots, \hat{q}_{i_{2}}, \hat{q}_{j}, \ldots, q_{7}\right) \wedge \\
& \prod_{\substack{j \neq i_{3} \\
j=i_{3}+1}}^{7} \Delta\left(q_{0}, \ldots, \hat{q}_{i_{3}}, \hat{q}_{j}, \ldots, q_{7}\right)-\prod_{\substack{j \neq i_{0} \\
j=i_{0}+1}}^{7} \Delta\left(q_{0}, \ldots, \hat{q}_{i_{0}}, \hat{q}_{j}, \ldots, q_{7}\right) \otimes \\
& {\left[r\left(q_{i_{0}}, q_{i_{1}}, q_{i_{2}}, q_{i_{3}} \mid q_{0}, \ldots, \hat{q}_{i_{0}}, \hat{q}_{i_{1}}, \hat{q}_{i_{2}}, \hat{q}_{i_{3}}, \ldots, q_{7}\right)\right]_{2} \otimes \prod_{\substack{j \neq i_{1} \\
j=i_{1}+1}}^{7} \Delta\left(q_{0}, \ldots, \hat{q}_{i_{1}}, \hat{q}_{j}, \ldots, q_{7}\right) \wedge} \\
& \prod_{\substack{j \neq i_{2} \\
j=i_{2}+1}}^{7} \Delta\left(q_{0}, \ldots, \hat{q}_{i_{2}}, \hat{q}_{j}, \ldots, q_{7}\right) \wedge \prod_{\substack{j \neq i_{3} \\
j=l i_{3}+1}}^{7} \Delta\left(q_{0}, \ldots, \hat{q}_{i_{3}}, \hat{q}_{j}, \ldots, q_{7}\right)+\prod_{\substack{j \neq i_{1} \\
j=i_{1}+1}}^{7} \Delta\left(q_{0}, \ldots, \hat{q}_{i_{1}}, \hat{q}_{j}, \ldots, q_{7}\right) \otimes \\
& {\left[r\left(q_{i_{0}}, q_{i_{1}}, q_{i_{2}}, q_{i_{3}} \mid q_{0}, \ldots, \hat{q}_{i_{0}}, \hat{q}_{i_{1}}, \hat{q}_{i_{2}}, \hat{q}_{i_{3}}, \ldots, q_{7}\right)\right]_{2} \otimes \prod_{\substack{j \neq i_{0} \\
j=i_{0}+1}}^{7} \Delta\left(q_{0}, \ldots, \hat{q}_{i_{0}}, \hat{q}_{j}, \ldots, q_{7}\right) \wedge} \\
& \prod_{\substack{j \neq i_{2} \\
j=i_{2}+1}}^{7} \Delta\left(q_{0}, \ldots, \hat{q}_{i_{2}}, \hat{q}_{j}, \ldots, q_{7}\right) \wedge \prod_{\substack{j \neq i_{3} \\
j=i_{3}+1}}^{7} \Delta\left(q_{0}, \ldots, \hat{q}_{i_{3}}, \hat{q}_{j}, \ldots, q_{7}\right)
\end{aligned}
$$




$$
\begin{aligned}
& -\prod_{\substack{j \neq i_{2} \\
j=i_{2}+1}}^{7} \Delta\left(q_{0}, \ldots, \hat{q}_{i_{2}}, \hat{q}_{j}, \ldots, q_{7}\right) \otimes\left[r\left(q_{i_{0}}, q_{i_{1}}, q_{i_{2}}, q_{i_{3}} \mid q_{0}, \ldots, \hat{q}_{i_{0}}, \hat{q}_{i_{1}}, \hat{q}_{i_{2}}, \hat{q}_{i_{3}}, \ldots, q_{7}\right)\right]_{2} \otimes \\
& \prod_{\substack{j \neq i_{0} \\
j=i_{0}+1}}^{7} \Delta\left(q_{0}, \ldots, \hat{q}_{i_{0}}, \hat{q}_{j}, \ldots, q_{7}\right) \wedge \prod_{\substack{j \neq i_{1} \\
j=i_{1}+1}}^{7} \Delta\left(q_{0}, \ldots, \hat{q}_{i_{2}}, \hat{q}_{2}, \ldots, q_{7}\right) \wedge \prod_{\substack{j \neq i_{3} \\
j=i_{3}+1}}^{7} \Delta\left(q_{0}, \ldots, \hat{q}_{i_{3}}, \hat{q}_{j}, \ldots, q_{7}\right) \\
& +\prod_{\substack{j \neq i_{3} \\
j=i_{3}+1}}^{7} \Delta\left(q_{0}, \ldots, \hat{q}_{i_{3}}, \hat{q}_{j}, \ldots, q_{7}\right) \otimes\left[r\left(q_{i_{0}}, q_{i_{1}}, q_{i_{2}}, q_{i_{3}} \mid q_{0}, \ldots, \hat{q}_{i_{0}}, \hat{q}_{i_{1}}, \hat{q}_{i_{2}}, \hat{q}_{i_{3}}, \ldots, q_{7}\right)\right]_{2} \otimes \\
& \left.\prod_{\substack{j \neq i_{0} \\
j=i_{0}+1}}^{7} \Delta\left(q_{0}, \ldots, \hat{q}_{i_{0}}, \hat{q}_{j}, \ldots, q_{7}\right) \wedge \prod_{\substack{j \neq i_{1} \\
j=i_{1}+1}}^{7} \Delta\left(q_{0}, \ldots, \hat{q}_{i_{1}}, \hat{q}_{j}, \ldots, q_{7}\right) \wedge \prod_{\substack{j \neq i_{2} \\
j=i_{2}+1}}^{7} \Delta\left(q_{0}, \ldots, \hat{q}_{i_{2}}, \hat{q}_{j}, \ldots, q_{7}\right)\right)
\end{aligned}
$$

$(\bmod 8)$

105 Lemma 14. $f_{0}^{6} \circ d=\partial \circ f_{1}^{6}$.

Proof. Let $\left(q_{0}, \ldots, q_{7}\right) \in G_{8}(6)$ on applying map $d$

$$
d\left(q_{0}, \ldots, q_{7}\right)=\sum_{i=0}^{7}(-1)^{i}\left(q_{0}, \ldots, \hat{q}_{i}, \ldots, q_{7}\right)
$$

Now apply map $f_{0}^{6}$

$$
\begin{aligned}
f_{0}^{6} \circ d\left(q_{0}, \ldots, q_{7}\right)= & \sum_{\substack{j \neq i \\
j=i+1}}^{7}(-1)^{j} \sum_{i=0}^{7}(-1)^{i} \triangle\left(q_{0}, \ldots, \hat{q}_{i}, \hat{q}_{j} \ldots, q_{7}\right) \otimes \frac{\Delta\left(q_{0}, \ldots, \hat{q}_{i+1}, \hat{q}_{j+1}, \ldots, q_{7}\right)}{\Delta\left(q_{0}, \ldots, \hat{q}_{i+2}, \hat{q}_{j+2}, \ldots, q_{7}\right)} \wedge \\
& \frac{\triangle\left(q_{0}, \ldots, \hat{q}_{i+2}, \hat{q}_{j+2}, \ldots, q_{7}\right)}{\triangle\left(q_{0}, \ldots, \hat{q}_{i+3}, \hat{q}_{j+3}, \ldots, q_{7}\right)} \wedge \frac{\Delta\left(q_{0}, \ldots, \hat{q}_{i+3}, \hat{q}_{j+3}, \ldots, q_{7}\right)}{\Delta\left(q_{0}, \ldots, \hat{q}_{i+4}, \hat{q}_{j+4}, \ldots, q_{7}\right)} \frac{\Delta\left(q_{0}, \ldots, \hat{q}_{i+4}, \hat{q}_{j+4}, \ldots, q_{7}\right)}{\Delta\left(q_{0}, \ldots, \hat{q}_{i+5}, \hat{q}_{j+5}, \ldots, q_{7}\right)} \wedge \\
& \frac{\triangle\left(q_{0}, \ldots, \hat{q}_{i+4}, \hat{q}_{j+5}, \ldots, q_{7}\right)}{\Delta\left(q_{0}, \ldots, \hat{q}_{i+6}, \hat{q}_{j+6}, \ldots, q_{7}\right)} \wedge \frac{\Delta\left(q_{0}, \ldots, \hat{q}_{i+6}, \hat{q}_{j+6}, \ldots, q_{7}\right)}{\Delta\left(q_{0}, \ldots, \hat{q}_{i+7}, \hat{q}_{j+7}, \ldots, q_{7}\right)}
\end{aligned}
$$

Apply $f_{1}^{6}$ on $\left(q_{0}, \ldots, q_{7}\right) \in G_{8}(6)$, then

$$
\begin{aligned}
& f_{1}^{6}\left(q_{0}, \ldots, q_{7}\right)=-\frac{1}{15}\left(\sum _ { \substack { i _ { 0 } = 0 \\
i _ { 1 } = i _ { 0 } + 1 \\
i _ { 2 } = i _ { 0 } + 2 \\
i _ { 3 } = i _ { 0 } + 1 } } ^ { 7 } ( - 1 ) ^ { i _ { 0 } } \left(\left\langle r\left(q_{i_{0}}, q_{i_{1}}, q_{i_{2}}, q_{i_{3}} \mid q_{0}, \ldots, \hat{q}_{i_{0}}, \hat{q}_{i_{1}}, \hat{q}_{i_{2}}, \hat{q}_{i_{3}}, \ldots, q_{7}\right)\right\rangle_{2} \otimes\right.\right. \\
& \prod_{\substack{j \neq i_{0} \\
j=i_{0}+1}}^{7} \Delta\left(q_{0}, \ldots, \hat{q}_{i_{0}}, \hat{q}_{j}, \ldots, q_{7}\right) \wedge \prod_{\substack{j \neq i_{1} \\
j=i_{1}+1}}^{7} \Delta\left(q_{0}, \ldots, \hat{q}_{i_{1}}, \hat{q}_{j}, \ldots, q_{7}\right) \wedge \prod_{\substack{j \neq i_{2} \\
j=i_{2}+1}}^{7} \Delta\left(q_{0}, \ldots, \hat{q}_{i_{2}}, \hat{q}_{j}, \ldots, q_{7}\right) \wedge \\
& \prod_{\substack{j \neq i_{3} \\
j=i_{3}+1}}^{7} \Delta\left(q_{0}, \ldots, \hat{q}_{i_{3}}, \hat{q}_{j}, \ldots, q_{7}\right)-\prod_{\substack{j \neq i_{0} \\
j=i_{0}+1}}^{7} \Delta\left(q_{0}, \ldots, \hat{q}_{i_{0}}, \hat{q}_{j}, \ldots, q_{7}\right) \otimes \\
& {\left[r\left(q_{i_{0}}, q_{i_{1}}, q_{i_{2}}, q_{i_{3}} \mid q_{0}, \ldots, \hat{q}_{i_{0}}, \hat{q}_{i_{1}}, \hat{q}_{i_{2}}, \hat{q}_{i_{3}}, \ldots, q_{7}\right)\right]_{2} \otimes \prod_{\substack{j \neq i_{1} \\
j=i_{1}+1}}^{7} \Delta\left(q_{0}, \ldots, \hat{q}_{i_{1}}, \hat{q}_{j}, \ldots, q_{7}\right) \wedge} \\
& \prod_{\substack{j \neq i_{2} \\
j=i_{2}+1}}^{7} \Delta\left(q_{0}, \ldots, \hat{q}_{i_{2}}, \hat{q}_{j}, \ldots, q_{7}\right) \wedge \prod_{\substack{j \neq i_{3} \\
j=l i_{3}+1}}^{7} \Delta\left(q_{0}, \ldots, \hat{q}_{i_{3}}, \hat{q}_{j}, \ldots, q_{7}\right)+\prod_{\substack{j \neq i_{1} \\
j=i_{1}+1}}^{7} \Delta\left(q_{0}, \ldots, \hat{q}_{i_{1}}, \hat{q}_{j}, \ldots, q_{7}\right) \otimes
\end{aligned}
$$




$$
\begin{aligned}
& {\left[r\left(q_{i_{0}}, q_{i_{1}}, q_{i_{2}}, q_{i_{3}} \mid q_{0}, \ldots, \hat{q}_{i_{0}}, \hat{q}_{i_{1}}, \hat{q}_{i_{2}}, \hat{q}_{i_{3}}, \ldots, q_{7}\right)\right]_{2} \otimes \prod_{\substack{j \neq i_{0} \\
j=i_{0}+1}}^{7} \Delta\left(q_{0}, \ldots, \hat{q}_{i_{0}}, \hat{q}_{j}, \ldots, q_{7}\right) \wedge} \\
& \prod_{\substack{j \neq i_{2} \\
j=i_{2}+1}}^{7} \Delta\left(q_{0}, \ldots, \hat{q}_{i_{2}}, \hat{q}_{j}, \ldots, q_{7}\right) \wedge \prod_{\substack{j \neq i_{3} \\
j=i_{3}+1}}^{7} \Delta\left(q_{0}, \ldots, \hat{q}_{i_{3}}, \hat{q}_{j}, \ldots, q_{7}\right) \\
& -\prod_{\substack{j \neq i_{2} \\
j=i_{2}+1}}^{7} \Delta\left(q_{0}, \ldots, \hat{q}_{i_{2}}, \hat{q}_{j}, \ldots, q_{7}\right) \otimes\left[r\left(q_{i_{0}}, q_{i_{1}}, q_{i_{2}}, q_{i_{3}} \mid q_{0}, \ldots, \hat{q}_{i_{0}}, \hat{q}_{i_{1}}, \hat{q}_{i_{2}}, \hat{q}_{i_{3}}, \ldots, q_{7}\right)\right]_{2} \otimes \\
& \prod_{\substack{j \neq i_{0} \\
j=i_{0}+1}}^{7} \Delta\left(q_{0}, \ldots, \hat{q}_{i_{0}}, \hat{q}_{j}, \ldots, q_{7}\right) \wedge \prod_{\substack{j \neq i_{1} \\
j=i_{1}+1}}^{7} \Delta\left(q_{0}, \ldots, \hat{q}_{i_{2}}, \hat{q}_{2}, \ldots, q_{7}\right) \wedge \prod_{\substack{j \neq i_{3} \\
j=i_{3}+1}}^{7} \Delta\left(q_{0}, \ldots, \hat{q}_{i_{3}}, \hat{q}_{j}, \ldots, q_{7}\right) \\
& +\prod_{\substack{j \neq i_{3} \\
j=i_{3}+1}}^{7} \Delta\left(q_{0}, \ldots, \hat{q}_{i_{3}}, \hat{q}_{j}, \ldots, q_{7}\right) \otimes\left[r\left(q_{i_{0}}, q_{i_{1}}, q_{i_{2}}, q_{i_{3}} \mid q_{0}, \ldots, \hat{q}_{i_{0}}, \hat{q}_{i_{1}}, \hat{q}_{i_{2}}, \hat{q}_{i_{3}}, \ldots, q_{7}\right)\right]_{2} \otimes \\
& \left.\prod_{\substack{j \neq i_{0} \\
j=i_{0}+1}}^{7} \Delta\left(q_{0}, \ldots, \hat{q}_{i_{0}}, \hat{q}_{j}, \ldots, q_{7}\right) \wedge \prod_{\substack{j \neq i_{1} \\
j=i_{1}+1}}^{7} \Delta\left(q_{0}, \ldots, \hat{q}_{i_{1}}, \hat{q}_{j}, \ldots, q_{7}\right) \wedge \prod_{\substack{j \neq i_{2} \\
j=i_{2}+1}}^{7} \Delta\left(q_{0}, \ldots, \hat{q}_{i_{2}}, \hat{q}_{j}, \ldots, q_{7}\right)\right)
\end{aligned}
$$

Apply map $\partial$ and all properties, then

$$
\begin{aligned}
\partial \circ f_{1}^{6}\left(q_{0}, \ldots, q_{7}\right)= & \sum_{\substack{j \neq i \\
j=i+1}}^{7}(-1)^{j} \sum_{i=0}^{7}(-1)^{i} \Delta\left(q_{0}, \ldots, \hat{q}_{i}, \hat{q}_{j} \ldots, q_{7}\right) \otimes \frac{\Delta\left(q_{0}, \ldots, \hat{q}_{i+1}, \hat{q}_{j+1}, \ldots, q_{7}\right)}{\Delta\left(q_{0}, \ldots, \hat{q}_{i+2}, \hat{q}_{j+2}, \ldots, q_{7}\right)} \wedge \\
& \frac{\Delta\left(q_{0}, \ldots, \hat{q}_{i+2}, \hat{q}_{j+2}, \ldots, q_{7}\right)}{\Delta\left(q_{0}, \ldots, \hat{q}_{i+3}, \hat{q}_{j+3}, \ldots, q_{7}\right)} \wedge \frac{\Delta\left(q_{0}, \ldots, \hat{q}_{i+3}, \hat{q}_{j+3}, \ldots, q_{7}\right)}{\Delta\left(q_{0}, \ldots, \hat{q}_{i+4}, \hat{q}_{j+4}, \ldots, q_{7}\right)} \frac{\Delta\left(q_{0}, \ldots, \hat{q}_{i+4}, \hat{q}_{j+4}, \ldots, q_{7}\right)}{\Delta\left(q_{0}, \ldots, \hat{q}_{i+5}, \hat{q}_{j+5}, \ldots, q_{7}\right)} \wedge \\
& \frac{\Delta\left(q_{0}, \ldots, \hat{q}_{i+4}, \hat{q}_{j+5}, \ldots, q_{7}\right)}{\Delta\left(q_{0}, \ldots, \hat{q}_{i+6}, \hat{q}_{j+6}, \ldots, q_{7}\right)} \wedge \frac{\Delta\left(q_{0}, \ldots, \hat{q}_{i+6}, \hat{q}_{j+6}, \ldots, q_{7}\right)}{\Delta\left(q_{0}, \ldots, \hat{q}_{i+7}, \hat{q}_{j+7}, \ldots, q_{7}\right)}
\end{aligned}
$$

\section{Generalized Geometry ( Weight $n=N$ )}

For generalization, construct the generalized diagram by connecting the two sub-complexes using generalized morphisms.

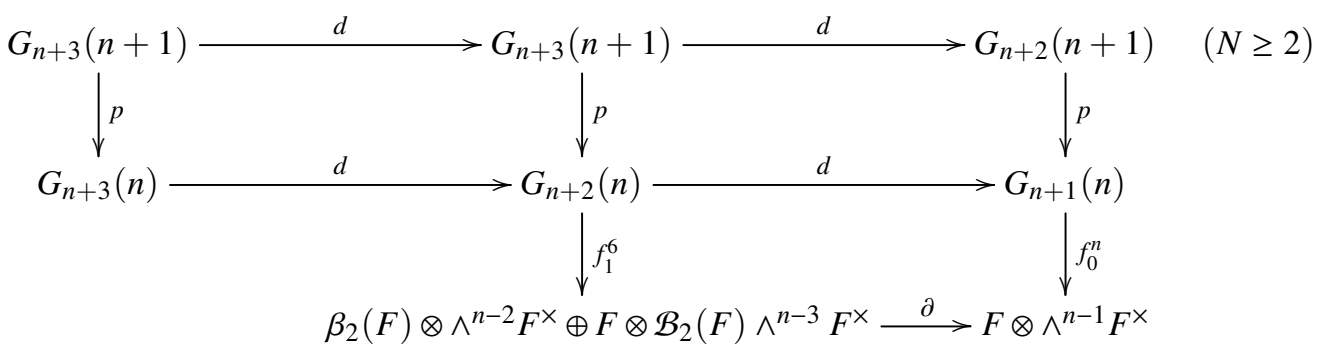

where

$$
f_{0}^{n}\left(q_{0}, \ldots, q_{n}\right)=\sum_{i=0}^{n}(-1)^{i} \triangle\left(q_{0}, \ldots, \hat{q}_{i}, \ldots, q_{n}\right) \otimes \frac{\Delta\left(q_{0}, \ldots, \hat{q}_{i+1}, \ldots, q_{n}\right)}{\Delta\left(q_{0}, \ldots, \hat{q}_{i+2}, \ldots, q_{n}\right)} \wedge \frac{\Delta\left(q_{0}, \ldots, \hat{q}_{i+2}, \ldots, q_{n}\right)}{\Delta\left(q_{0}, \ldots, \hat{q}_{i+3}, \ldots, q_{n}\right)} \wedge
$$




$$
\frac{\triangle\left(q_{0}, \ldots, \hat{q}_{i+3}, \ldots, q_{n}\right)}{\triangle\left(q_{0}, \ldots, \hat{q}_{i+4}, \ldots, q_{n}\right)} \wedge \ldots \wedge \frac{\Delta\left(q_{0}, \ldots, \hat{q}_{i+n-2}, \ldots, q_{n}\right)}{\triangle\left(q_{0}, \ldots, \hat{q}_{i+n-1}, \ldots, q_{n}\right)} \wedge \frac{\Delta\left(q_{0}, \ldots, \hat{q}_{i+n-1}, \ldots, q_{n}\right)}{\triangle\left(q_{0}, \ldots, \hat{q}_{i+n}, \ldots, q_{n}\right)} \quad(\bmod \mathrm{n}+1)
$$

and

$$
\begin{aligned}
& f_{1}^{n}\left(q_{0}, \ldots, q_{n+1}\right)=(-1)^{N} \frac{1}{{ }^{n} C_{2}}\left(\sum _ { \substack { i _ { 0 } = 0 \\
i _ { 1 } = i _ { 0 } + 1 \\
i _ { 2 } = i _ { 0 } + 2 } } ^ { n + 1 } ( - 1 ) ^ { i _ { 0 } } \left(\left\langle r\left(q_{i_{0}}, \ldots, q_{i_{n-3}} \mid q_{0}, \ldots, \hat{q}_{i_{0}}, \ldots, \hat{q}_{i_{n-3}}, \ldots, q_{n+1}\right)\right\rangle_{2} \otimes\right.\right. \\
& i_{n-3}=\dot{i_{0}}+n-3 \\
& \prod_{\substack{j \neq i_{0} \\
j=i_{0}+1}}^{n+1} \Delta\left(q_{0}, \ldots, \hat{q}_{i_{0}}, \hat{q}_{j}, \ldots, q_{n+1}\right) \wedge \prod_{\substack{j \neq i_{1} \\
j=i_{1}+1}}^{n+1} \Delta\left(q_{0}, \ldots, \hat{q}_{i_{1}}, \hat{q}_{j}, \ldots, q_{n+1}\right) \wedge \ldots \wedge \\
& \prod_{\substack{j \neq i_{n-3} \\
m j=i_{n-3}+1}}^{n+1} \Delta\left(q_{0}, \ldots, \hat{q}_{i_{n-3}}, \hat{q}_{j}, \ldots, q_{n+1}\right)-\prod_{\substack{j \neq i_{0} \\
j=i_{0}+1}}^{n+1} \Delta\left(q_{0}, \ldots, \hat{q}_{i_{0}}, \hat{q}_{j}, \ldots, q_{n+1}\right) \otimes \\
& {\left[r\left(q_{i_{0}}, \ldots, q_{i_{n-3}} \mid q_{0}, \ldots, \hat{q}_{i_{0}}, \ldots, \hat{q}_{i_{n-3}}, \ldots, q_{n+1}\right)\right]_{2} \otimes \prod_{\substack{j \neq i_{1} \\
j=i_{1}+1}}^{n+1} \Delta\left(q_{0}, \ldots, \hat{q}_{i_{1}}, \hat{q}_{j}, \ldots, q_{n+1}\right)} \\
& \wedge \ldots \wedge \prod_{\substack{j \neq i_{n-3} \\
j=1+i_{n-3}}}^{n+1} \Delta\left(q_{0}, \ldots, \hat{q}_{i_{n-3}}, \hat{q}_{j}, \ldots, q_{n+1}\right)+ \\
& (-1)^{n+1} \prod_{\substack{j \neq i_{n-3} \\
j=1+i_{n-3}}}^{n+1} \Delta\left(q_{0}, \ldots, \hat{q}_{i_{n-3}}, \hat{q}_{j}, \ldots q_{n+1}\right) \otimes\left[r \left(q_{i_{0}}, \ldots, q_{i_{n-3}} \mid q_{0}, \ldots, \hat{q}_{i_{0}}, \ldots, \hat{q}_{i_{n-3}}, \ldots\right.\right. \\
& \left.\left.q_{n+1}\right)\right]_{2} \otimes \prod_{\substack{j \neq i_{0} \\
j=i_{0}+1}}^{n+1} \Delta\left(q_{0}, \ldots, \hat{q}_{i_{0}}, \hat{q}_{j}, \ldots, q_{n+1}\right) \wedge \ldots \wedge \prod_{\substack{j \neq i_{n-2} \\
j=1+i_{n-1}}}^{n+1} \Delta\left(q_{0}, \ldots, \hat{q}_{i_{n-2}}, \hat{q}_{j}, \ldots, q_{n+1}\right) \quad(\bmod \mathrm{n}+2)
\end{aligned}
$$

Theorem 1. The lower square of the generalized diagram I is commutative.

Proof. Let $\left(q_{0}, \ldots, q_{n+1}\right) \in G_{n+2}(n)$ and apply map $d$, then

$$
d\left(q_{0}, \ldots, q_{n+1}\right)=\sum_{i=0}^{n+1}(-1)^{i}\left(q_{0}, \ldots, \hat{q}_{i}, \ldots, q_{n+1}\right)
$$

Apply map $f_{0}^{n}$ on $d\left(q_{0}, \ldots, q_{n+1}\right)$, then

$$
\begin{aligned}
f_{0}^{n} \circ d\left(q_{0}, \ldots, q_{n+1}\right)= & \sum_{j=i+1}^{n+1}(-1)^{j} \sum_{i=0}^{n+1}(-1)^{i} \Delta\left(q_{0}, \ldots, \hat{q}_{i}, \hat{q}_{j}, \ldots, q_{n+1}\right) \otimes \frac{\Delta\left(q_{0}, \ldots, \hat{q}_{i+1}, \hat{q}_{j+1}, \ldots, q_{n+1}\right)}{\Delta\left(q_{0}, \ldots, \hat{q}_{i+2}, \hat{q}_{j+2}, \ldots, q_{n+1}\right)} \wedge \\
& \frac{\Delta\left(q_{0}, \ldots, \hat{q}_{i+2}, \hat{q}_{j+2}, \ldots, q_{n+1}\right)}{\Delta\left(q_{0}, \ldots, \hat{q}_{i+3}, \hat{q}_{j+3}, \ldots, q_{n+1}\right)} \wedge \frac{\Delta\left(q_{0}, \ldots, \hat{q}_{i+3}, \hat{q}_{j+3}, \ldots, q_{n+1}\right)}{\Delta\left(q_{0}, \ldots, \hat{q}_{i+4}, \hat{q}_{j+4}, \ldots, q_{n+1}\right)} \wedge \ldots \wedge
\end{aligned}
$$




$$
\frac{\Delta\left(q_{0}, \ldots, \hat{q}_{i+n-1}, \hat{q}_{j+n-1}, \ldots, q_{n+1}\right)}{\triangle\left(q_{0}, \ldots, \hat{q}_{i+n}, \hat{q}_{j+n}, \ldots, q_{n+1}\right)} \wedge \frac{\Delta\left(q_{0}, \ldots, \hat{q}_{i+n}, \hat{q}_{j+n}, \ldots, q_{n+1}\right)}{\Delta\left(q_{0}, \ldots, \hat{q}_{i+n+1}, \hat{q}_{j+n+1}, \ldots, q_{n+1}\right)}
$$

Apply morphism $f_{1}^{n}$ on $\left(q_{0}, \ldots, q_{n+1}\right) \in G_{n+2}(n)$, then

$$
\begin{aligned}
& f_{1}^{n}\left(q_{0}, \ldots, q_{n+1}\right)=(-1)^{N} \frac{1}{{ }^{n} C_{2}}\left(\sum _ { \substack { i _ { 0 } = 0 \\
i _ { 1 } = i _ { 0 } + 1 \\
i _ { 2 } = i _ { 0 } + 2 } } ^ { n + 1 } ( - 1 ) ^ { i _ { 0 } } \left(\left\langle r\left(q_{i_{0}}, \ldots, q_{i_{n-3}} \mid q_{0}, \ldots, \hat{q}_{i_{0}}, \ldots, \hat{q}_{i_{n-3}}, \ldots, q_{n+1}\right)\right\rangle_{2} \otimes\right.\right. \\
& i_{n-3}=\dot{i_{0}}+n-3 \\
& \prod_{\substack{j \neq i_{0} \\
j=i_{0}+1}}^{n+1} \Delta\left(q_{0}, \ldots, \hat{q}_{i_{0}}, \hat{q}_{j}, \ldots, q_{n+1}\right) \wedge \prod_{\substack{j \neq i_{1} \\
j=i_{1}+1}}^{n+1} \Delta\left(q_{0}, \ldots, \hat{q}_{i_{1}}, \hat{q}_{j}, \ldots, q_{n+1}\right) \wedge \ldots \wedge \\
& \prod_{\substack{j \neq i_{n-3} \\
j=i_{n-3}+1}}^{n+1} \Delta\left(q_{0}, \ldots, \hat{q}_{i_{n-3}}, \hat{q}_{j}, \ldots, q_{n+1}\right)-\prod_{\substack{j \neq i_{0} \\
j=i_{0}+1}}^{n+1} \Delta\left(q_{0}, \ldots, \hat{q}_{i_{0}}, \hat{q}_{j}, \ldots, q_{n+1}\right) \otimes \\
& {\left[r\left(q_{i_{0}}, \ldots, q_{i_{n-3}} \mid q_{0}, \ldots, \hat{q}_{i_{0}}, \ldots, \hat{q}_{i_{n-3}}, \ldots, q_{n+1}\right)\right]_{2} \otimes \prod_{\substack{j \neq i_{1} \\
j=i_{1}+1}}^{n+1} \Delta\left(q_{0}, \ldots, \hat{q}_{i_{1}}, \hat{q}_{j}, \ldots, q_{n+1}\right)} \\
& \wedge \ldots \wedge \prod_{\substack{j \neq i_{n-3} \\
j=1+i_{n-3}}}^{n+1} \Delta\left(q_{0}, \ldots, \hat{q}_{i_{n-3}}, \hat{q}_{j}, \ldots, q_{n+1}\right)+ \\
& (-1)^{n+1} \prod_{\substack{j \neq i_{n-3} \\
j=1+i_{n-3}}}^{n+1} \Delta\left(q_{0}, \ldots, \hat{q}_{i_{n-3}}, \hat{q}_{j}, \ldots q_{n+1}\right) \otimes\left[r \left(q_{i_{0}}, \ldots, q_{i_{n-3}} \mid q_{0}, \ldots, \hat{q}_{i_{0}}, \ldots, \hat{q}_{i_{n-3}}, \ldots\right.\right. \\
& \left.\left.q_{n+1}\right)\right]_{2} \otimes \prod_{\substack{j \neq i_{0} \\
j=i_{0}+1}}^{n+1} \Delta\left(q_{0}, \ldots, \hat{q}_{i_{0}}, \hat{q}_{j}, \ldots, q_{n+1}\right) \wedge \ldots \wedge \prod_{\substack{j \neq i_{n-2} \\
j=1+i_{n-1}}}^{n+1} \Delta\left(q_{0}, \ldots, \hat{q}_{i_{n-2}}, \hat{q}_{j}\right. \\
& \left.\left.\ldots, q_{n+1}\right)\right) \quad(\bmod n+2)
\end{aligned}
$$

apply map $\partial$, it becomes

$$
\begin{gathered}
\partial \circ f_{1}^{n}\left(q_{0}, \ldots, q_{n+1}\right)=(-1)^{N} \frac{1}{{ }^{n} C_{2}}\left(\sum _ { \substack { i _ { 0 } = 0 \\
i _ { 1 } = i _ { 0 } + 1 \\
i _ { 2 } = i _ { 0 } + 2 \\
\vdots \\
i _ { n - 3 } = i _ { 0 } + n - 3 } } ^ { n + 1 } ( - 1 ) ^ { i _ { 0 } } \left(r\left(q_{i_{0}}, \ldots, q_{i_{n-3}} \mid q_{0}, \ldots, \hat{q}_{i_{0}}, \ldots, \hat{q}_{i_{n-3}}, \ldots, q_{n+1}\right) \otimes\right.\right. \\
\quad\left(1-r\left(q_{i_{0}}, \ldots, q_{i_{n-3}} \mid q_{0}, \ldots, \hat{q}_{i_{0}}, \ldots, \hat{q}_{i_{n-3}}, \ldots, q_{n+1}\right) \wedge \prod_{j \neq i_{0}}^{n+1} \Delta\left(q_{0}, \ldots, \hat{q}_{i_{0}}, \hat{q}_{j}, \ldots, q_{n+1}\right)\right. \\
\wedge \prod_{j=i_{0}}^{n+1} \Delta\left(q_{0}, \ldots, \hat{q}_{i_{1}}, \hat{q}_{j}, \ldots, q_{n+1}\right) \wedge \ldots \wedge \prod_{j=i_{1}+1}^{n+1} \Delta \prod_{j=i_{n-3}}^{n=i_{n-3}+1}
\end{gathered}
$$




$$
\begin{aligned}
& \left(1-r\left(q_{i_{0}}, \ldots, q_{i_{n-3}} \mid q_{0}, \ldots, \hat{q}_{i_{0}}, \ldots, \hat{q}_{i_{n-3}}, \ldots, q_{n+1}\right)\right) \otimes r\left(q_{i_{0}}, \ldots, q_{i_{n-3}} \mid q_{0}, \ldots, \hat{q}_{i_{0}}, \ldots, \hat{q}_{i_{n-3}}, \ldots\right. \\
& \left.q_{n+1}\right) \wedge \prod_{\substack{j \neq i_{0} \\
j=i_{0}+1}}^{n+1} \Delta\left(q_{0}, \ldots, \hat{q}_{i_{0}}, \hat{q}_{j}, \ldots, q_{n+1}\right) \wedge \prod_{\substack{j \neq i_{1} \\
j=i_{1}+1}}^{n+1} \Delta\left(q_{0}, \ldots, \hat{q}_{i_{1}}, \hat{q}_{j}, \ldots, q_{n+1}\right) \wedge \ldots \wedge \\
& \prod_{\substack{j \neq i_{n-3} \\
j=i_{n-3}+1}}^{n+1} \Delta\left(q_{0}, \ldots, \hat{q}_{i_{n-3}}, \hat{q}_{j}, \ldots, q_{n+1}\right)-\prod_{\substack{j \neq i_{1} \\
j=i_{1}+1}}^{n+1} \Delta\left(q_{0}, \ldots, \hat{q}_{i_{1}}, \hat{q}_{j}, \ldots, q_{n+1}\right) \otimes \\
& \left(1-r\left(q_{i_{0}}, \ldots, q_{i_{n-3}} \mid q_{0}, \ldots, \hat{q}_{i_{0}}, \ldots, \hat{q}_{i_{n-3}}, \ldots, q_{n+1}\right)\right) \wedge r\left(q_{i_{0}}, \ldots, q_{i_{n-3}} \mid q_{0}, \ldots, \hat{q}_{i_{0}}, \ldots, \hat{q}_{i_{n-3}}, \ldots,\right. \\
& \left.q_{n+1}\right) \\
& \wedge \prod_{\substack{j \neq i_{0} \\
j=i_{0}+1}}^{n+1} \Delta\left(q_{0}, \ldots, \hat{q}_{i_{0}}, \hat{q}_{j}, \ldots, q_{n+1}\right) \wedge \ldots \wedge \prod_{\substack{j \neq i_{n-3} \\
j=1+i_{n-3}}}^{n+1} \Delta\left(q_{0}, \ldots, \hat{q}_{i_{n-3}}, \hat{q}_{j}, \ldots, q_{n+1}\right) \\
& + \\
& (-1)^{n+1} \prod_{\substack{j \neq i_{n-3} \\
j=1+i_{n-3}}}^{n+1} \Delta\left(q_{0}, \ldots, \hat{q}_{i_{n-3}}, \hat{q}_{j}, \ldots q_{n+1}\right) \otimes\left(1-r\left(q_{i_{0}}, \ldots, q_{i_{n-3}} \mid q_{0}, \ldots, \hat{q}_{i_{0}}, \ldots, \hat{q}_{i_{n-3}}, \ldots\right.\right. \\
& \left.\left.\left., q_{n+1}\right)\right) \wedge r\left(q_{i_{0}}, \ldots, q_{i_{n-3}} \mid q_{0}, \ldots, \hat{q}_{i_{0}}, \ldots, \hat{q}_{i_{n-3}}, \ldots, q_{n+1}\right)\right) \wedge \prod_{\substack{j \neq i_{0} \\
j=i_{0}+1}}^{n+1} \Delta\left(q_{0}, \ldots, \hat{q}_{i_{0}}, \hat{q}_{j}, \ldots, q_{n+1}\right) \\
& \left.\wedge \ldots \wedge \prod_{\substack{j \neq i_{n-2} \\
j=1+i_{n-2}}}^{n+1} \Delta\left(q_{0}, \ldots, \hat{q}_{i_{n-2}}, \hat{q}_{j}, \ldots, q_{n+1}\right)\right)
\end{aligned}
$$

now apply all properties of wedge, tensor, Siegel and dummy indices, it becomes

$$
\begin{aligned}
\partial \circ f_{1}^{n}\left(q_{0}, \ldots, q_{n+1}\right)= & \sum_{j=i+1}^{n+1}(-1)^{j} \sum_{i=0}^{n+1}(-1)^{i} \triangle\left(q_{0}, \ldots, \hat{q}_{i}, \hat{q}_{j}, \ldots, q_{n+1}\right) \otimes \frac{\Delta\left(q_{0}, \ldots, \hat{q}_{i+1}, \hat{q}_{j+1}, \ldots, q_{n+1}\right)}{\Delta\left(q_{0}, \ldots, \hat{q}_{i+2}, \hat{q}_{j+2}, \ldots, q_{n+1}\right)} \wedge \\
& \frac{\Delta\left(q_{0}, \ldots, \hat{q}_{i+2}, \hat{q}_{j+2}, \ldots, q_{n+1}\right)}{\Delta\left(q_{0}, \ldots, \hat{q}_{i+3}, \hat{q}_{j+3}, \ldots, q_{n+1}\right)} \wedge \frac{\triangle\left(q_{0}, \ldots, \hat{q}_{i+3}, \hat{q}_{j+3}, \ldots, q_{n+1}\right)}{\triangle\left(q_{0}, \ldots, \hat{q}_{i+4}, \hat{q}_{j+4}, \ldots, q_{n+1}\right)} \wedge \ldots \wedge \\
& \frac{\Delta\left(q_{0}, \ldots, \hat{q}_{i+n-1}, \hat{q}_{j+n-1}, \ldots, q_{n+1}\right)}{\Delta\left(q_{0}, \ldots, \hat{q}_{i+n}, \hat{q}_{j+n}, \ldots, q_{n+1}\right)} \wedge \frac{\Delta\left(q_{0}, \ldots, \hat{q}_{i+n}, \hat{q}_{j+n}, \ldots, q_{n+1}\right)}{\Delta\left(q_{0}, \ldots, \hat{q}_{i+n+1}, \hat{q}_{j+n+1}, \ldots, q_{n+1}\right)}
\end{aligned}
$$

So from Eq.(53) and Eq.(56) and using dummy indices, theorem 1 is hence proved.

\section{Conclusion}

In this study the generalization of morphisms $f_{0}^{n}$ and $f_{1}^{n}$ are presented to connect Cathelineau infinitesimal and Grassmannian chain complexes for generalized geometry. This work will play significant role in the fields of Algebraic K-theory, Chain complexes, Algebraic Topology, Homological Algebra and Polylogarithmic group theory.

Conflicts of Interest: The authors declare no conflict of interest.

\section{References}

1. Suslin, A.A. Homology of $G L_{n}$, characteristic classes and Milnor's K-theory, in: Proceedings of the Steklov Institute of Mathematics 1985, Lecture Notes in Mathematics (1046), Springer-Verlag, New York, USA, 1989;, 207-226. 
2. Goncharov, A.B. Geometry of configuration, polylogarithms and motivic cohomology, Advances in Mathematics, 1995, 114(2), 197-318. http://dx.doi.org/10.1006/aima.1995.1045.

3. Goncharov, A.B. Eucledian scissor congruence groups and mixed tate motives over dual numbers, Mathematical Research Letters, 2004, 11, 771-784.

4. Goncharov, A.B.; Zhao,J. Grassmannian trilogarithm, Compositio Mathematica, 2001, 127, 83-108.

5. Cathelineau, J.L. Remarques sur les Différentielles des Polylogarithmes Uniformes, Ann. Inst. Fourier, Grenoble, 1996, 46, 1327-1347.

6. Cathelineau, J.L. Infinitesimal Polylogarithms, multiplicative presentation of Kähler Differential and Goncharov Complexes, Talk at the workshop on polylogarthms, Essen, 1997; May 1-4

7. Cathelineau, J.L. The tangent complex to the Bloch-Suslin complex, Bull. Soc. Math. France, 2007, 135, 565-597.

8. Siddiqui, R. Morphism between classical and infinitesimal polylogarithmic and grassmannian complexes, International Journal of Algebra, 2012, 6(22), 1087-1096.

9. Hussain, S.; Siddiqui, R. Grassmannian Complex and Second Order Tangential Complex, Punjab University Journal of Mathematics, 2016, 48(02), 91-111.

10. Khalid,M.; Javed, K.; Azhar, I. New Homomorphism between Grassmannian and Infinitesimal Complexes, International Journal of Algebra, 2016, 10(3), 97-112. http:// dx.doi.org /10.12988 /ija.2016.6213.

11. Khalid,M.; Javed, K.; Azhar, I. Generalization of Grassmannian and polylogarithmatic groups complex , International Journal of Algebra, 2016, 10(5), 221-237. http://dx.doi.org/10.12988 / ija.2016.6323.

12. Khalid,M.; Javed, K.; Azhar, I. Higher Order Grassmannian Complexes, International Journal of Algebra, 2016, 10(09), 405-413. http://dx.doi.org/10.12988/ija.2016.6640.

13. Khalid,M.; Javed, K.; Azhar, I. Generalization of Higher Order Homomorphism in Configuration Complexes, Punjab University Journal of Mathematics, 2017, 49(1), Accepted

14. Elbaz-Vincent, P.; Gangl, H. On Poly(ana)logs I, Compositio Mathematica, 2002, 130, 161-210.

15. Siegel, C.L. Approximation algebraischer zahlen, Mathem.Ze/tschr. 1921, 10, 173-213

(C) 2017 by the authors. Licensee Preprints, Basel, Switzerland. This article is an open access article distributed under the terms and conditions of the Creative Commons by Attribution (CC-BY) license (http://creativecommons.org/licenses/by/4.0/). 